\title{
Reddening and the Extinction Law at High Galactic Latitude
}

Kristen A. Larson

Western Washington University, kristen.larson@wwu.edu

D. C. B. Whittet

Follow this and additional works at: https://cedar.wwu.edu/physicsastronomy_facpubs

Part of the Astrophysics and Astronomy Commons

\section{Recommended Citation}

Larson, Kristen A. and Whittet, D. C. B., "Reddening and the Extinction Law at High Galactic Latitude" (2005). Physics \& Astronomy. 2 .

https://cedar.wwu.edu/physicsastronomy_facpubs/2 


\title{
REDDENING AND THE EXTINCTION LAW AT HIGH GALACTIC LATITUDE
}

\author{
K. A. LARSON \\ Department of Physics and Astronomy, Western Washington University, Bellingham, WA 98225 \\ AND \\ D. C. B. WhitTet \\ Department of Physics, Applied Physics and Astronomy, Rensselaer Polytechnic Institute, Troy, NY 12180 \\ Received 2004 October 16; accepted 2004 December 16
}

\begin{abstract}
We present near-infrared (JHKL) photometry of 103 southern stars located behind translucent interstellar clouds at high Galactic latitude. Our data are combined with visual photometry and spectral type information from the literature in a detailed analysis of the wavelength dependence of interstellar extinction by dust in these high-latitude clouds. We investigate the shape of the near-infrared extinction curve and evaluate the total line-of-sight extinction $\left(A_{V}\right)$ and ratio of total-to-selective extinction $\left(R_{V}\right)$ in each line of sight. Sources of uncertainty in $R_{V}$ arising from photometric errors and spectral classification errors are carefully assessed and quantified as functions of the line-ofsight reddening. We detect appreciable differences in our results compared with the average extinction curve for dust in the diffuse interstellar medium (ISM) close to the Galactic plane. Assuming a power-law form in the nearinfrared, we find the mean for high-latitude clouds to be well described by $\left\langle A_{\lambda}\right\rangle \propto \lambda^{-2.3}$, somewhat steeper than that for the diffuse ISM $\left(\left\langle A_{\lambda}\right\rangle \propto \lambda^{-1.8}\right)$. Our sample includes a substantial number of lines of sight with low $R_{V}$ values ( $47 \%$ with $R_{V}<2.8$ ) relative to the diffuse ISM average of $3.05 \pm 0.15$. We conclude that many high-latitude clouds have enhanced abundances of relatively small grains.
\end{abstract}

Subject headings: dust, extinction — infrared: ISM — ISM: clouds

Online material: machine-readable tables

\section{INTRODUCTION}

The molecular component of the cold interstellar medium (ISM) has a local scale height of about $75 \mathrm{pc}$ away from the midplane of the Milky Way, a factor of 2 thinner than the atomic ISM component and late-type stellar population. Until the 1980s, most studies of the molecular component of the ISM were confined to low Galactic latitudes, including star-forming regions relatively near the Galactic midplane such as those in Taurus and Orion. More recently, greater sensitivity of radio telescopes and analysis of all-sky infrared surveys led to the discovery and study of tenuous, low-mass clouds located at high Galactic latitudes away from the midplane. Almost all of the early searches for molecular gas in high-latitude clouds used the associated dust to identify candidate regions. Surveys by Magnani et al. (1985), Hartley et al. (1986), and others achieved this by searching photographic plates for extinction and reddening of background starlight as well as scattering of diffuse light reflected from the Galactic disk. Their subsequent observations detected $\mathrm{CO}(J=1-0)$ at $T_{A}>0.3 \mathrm{~K}$ in about one-quarter of the candidate positions. High-latitude clouds are nearby, with an average distance of about $100 \mathrm{pc}$, assuming an altitude equal to the expectation value for the cold ISM's distribution away from the midplane. Proximity of the clouds is confirmed by CO line velocities near $v_{\text {LSR }}$. Some of the molecular clouds at high latitude are dark, such as those in the Lynds compilation. Other high-latitude clouds in which $\mathrm{CO}$ is detected are translucent, with about $1 \mathrm{mag}$ of visual extinction on average and a wide variety of morphologies. Some high-latitude clouds exhibit rich velocity structure, but many of the high-latitude clouds in which $\mathrm{CO}$ is detected are quiescent with narrow line widths and less than $1 \mathrm{~km} \mathrm{~s}^{-1}$ of internal dispersion.

To extend the targeted searches, the northern and southern hemispheres of the high-latitude sky have more recently been sampled for CO emission at $1^{\circ}$ spacing by Hartmann et al. (1998) and Magnani et al. (2000), respectively, yielding several new detections but missing some of the small high-latitude molecular clouds previously identified. Overall, it is estimated that a few percent of high-latitude lines of sight pass through a molecular cloud, with a higher surface filling fraction in the southern hemisphere than the northern hemisphere (Magnani et al. 2000). A complete inventory of high-latitude $\mathrm{CO}$ still does not exist, however, because most surveys for $\mathrm{CO}$ use subjective criteria to select target regions and because many regions in which $\mathrm{CO}$ is detected have never been fully mapped. Even if a complete map of $\mathrm{CO}$ existed, the conversion from $N(\mathrm{CO})$ to total molecular abundance would first be complicated by the fact that the $\mathrm{CO}$ to $\mathrm{H}_{2}$ conversion factor is not well determined. Magnani \& Onello (1995) give a detailed discussion of the $X(\mathrm{CO})$ variation in the translucent ISM. Many high-latitude translucent clouds are located far from strong UV radiation sources and are self-shielding clouds with high relative $\mathrm{CO}$ abundance, called "CO-rich" (van Dishoeck 1994). In other high-latitude objects, however, molecular hydrogen is nearly as abundant as atomic hydrogen even though $\mathrm{CO}$ abundance is below detectable levels (Reach et al. 1994).

In the absence of a complete CO map, several important attempts have been made to infer all-sky maps of molecular hydrogen gas. Analyzing high-latitude structures in the IRAS $100 \mu \mathrm{m}$ maps, Désert et al. (1988) identified regions of excess infrared emission above the infrared emission expected from local correlation with atomic hydrogen. These infrared-excess regions are attributed to heated dust associated with molecular hydrogen and include objects where CO is not detected. Reach et al. (1998) identified and mapped high-latitude infrared excess with more recent data from COBE DIRBE and the Leiden-Dwingeloo atomic hydrogen survey. While it is possible to identify high-latitude 
molecular clouds with this method, converting maps to extinction has proven to be challenging. The difficulty presented by variation in the molecular fraction is compounded by variation in the dust-to-gas ratio and the shape of the extinction curve, implied by global variations in the local value of the far-infrared to atomic hydrogen ratio. Even if extinction due to dust is calculated directly from the thermal emission of dust, the reddening per unit flux density of $100 \mu \mathrm{m}$ emission must be known. The oft-used Burstein \& Heiles (1982) map of reddening was more recently replaced by that of Schlegel et al. (1998), who used a composite map of the COBE DIRBE and IRAS Sky Survey Atlas data, the Leiden-Dwingeloo atomic hydrogen data, and the colors of elliptical galaxies to calibrate their reddening map. Although the newer map uses infrared data and more recent atomic hydrogen data, there is some evidence that the map is not accurate for visual extinctions greater than 0.5 mag (Arce \& Goodman 1999). It is therefore more important than ever to study high-latitude extinction by dust directly.

While the dust in high-latitude clouds was instrumental in their discovery and must play an important role in their gas chemistry, little information exists about the dust content of the high-latitude clouds. The goal of our project is to characterize the dust in sight lines through a variety of high-latitude clouds by observing photometric extinction toward background stars. We analyze near-infrared (NIR) color excess to measure the amount of extinction in each line of sight and to measure the normalized extinction law in the infrared. Among the ways to characterize normalized extinction, we focus on the ratio of extinction to reddening, $R_{V}$. This parameter is not only required to convert blue visual reddening to visual extinction, it also characterizes the size distribution of the dust and the shape of the entire extinction curve beyond the visible (Cardelli et al. 1989). The ratio $R_{V}$ is an important parameter for understanding molecule formation on grain surfaces and shielding in the high-latitude ISM and therefore is necessary to convert any molecular spectral signature to extinction. We present the photometric data in $\S 2$ and parameterization of the extinction curves in $\S 3$. Because of the importance of $R_{V}$ and the new opportunities that the Two Micron All Sky Survey (2MASS) survey provides to measure it, we also carefully consider the uncertainty in $R_{V}$ when it is measured from color excess, including uncertainties due to error in spectral type. In $\S 4$ we conclude with a discussion of variations in normalized extinction curves and our results at high latitude.

\section{PHOTOMETRY AND REDDENING}

Of the 105 lines of sight analyzed in this paper, 90 were chosen from a study of optical photometry and spectroscopy of early-type stars in the vicinity of high-latitude $100 \mu \mathrm{m}$ emission by Penprase (1992). We selected stars reported by Penprase (1992) to have reddening $E_{B-V}>0.10 \mathrm{mag}$ that are located within the observing range of southern hemisphere telescopes used in our project. High Galactic latitude can be defined as $|b|>20^{\circ}$. Some of the stars observed by Penprase (1992) are located at $10^{\circ}<|b|<20^{\circ}$ behind molecular clouds that are likely to be similar to clouds found at higher latitudes. The star HD 210121 was added on the basis of the conclusions of Welty \& Fowler (1992), and the star HD 203532 was added to the program from the NIR study of Whittet \& van Breda (1980). The remaining stars were added by using the SIMBAD database to find reddened stars in the vicinity of sight lines already in the program. These last stars are typically later spectral types than stars in Penprase (1992).

Photometry, spectral type, and appropriate intrinsic colors are necessary to measure the color excess with respect to a reference passband. The reference passband is often chosen to be the visual, such that reddening is defined as

$$
E_{\lambda-V}=m_{\lambda}-V-\left(m_{\lambda}-V\right)_{0} .
$$

NIR photometry of program stars was obtained at the South African Astronomical Observatory (SAAO) on 1994 July 1522, 1995 April 12-17, 1996 January 9-15, 1997 May 20-22, and 1997 October 7-13. The Mark III InSb Infrared Photometer was used on the $1.9 \mathrm{~m}$ telescope. Most stars were observed with an $18^{\prime \prime}$ aperture and a $30^{\prime \prime}$ north-south chop for sky subtraction; a few stars in more crowded fields were observed with a $12^{\prime \prime}$ aperture. Results are reduced to the photometric system of Carter (1990), giving magnitudes in standard $J(1.25 \mu \mathrm{m}), H(1.65 \mu \mathrm{m})$, $K(2.2 \mu \mathrm{m})$, and $L(3.5 \mu \mathrm{m})$ passbands. Standard uncertainty in these passbands is $\sigma_{J}=0.02 \mathrm{mag}, \sigma_{H}=0.02 \mathrm{mag}, \sigma_{K}=$ $0.03 \mathrm{mag}$, and $\sigma_{L}=0.05 \mathrm{mag}$. Nights are initially reduced with constant zero points. If trends in standard star residuals on any night are larger than these uncertainties, data on that night are separated into two or three subsets and reduced again with separate zero points. No evidence for stellar variability is found in the data for stars observed on multiple nights. Data from multiple nights are then averaged, weighted by errors that include the error in mean zero point. The resulting NIR magnitudes and uncertainties are listed in columns (2)-(9) of Table 1. Previously published data are noted.

Optical photometric data for program stars are primarily from the Mermilliod \& Mermilliod (1994) compilation of $U B V$ photometry, which includes the Penprase (1992) photometric data set. For stars without multiple references in the Mermilliod \& Mermilliod (1994) compilation, photometry is selected directly from Penprase (1992) and the SIMBAD database. In a few cases, we use new optical photometry obtained at SAAO (D. Kilkenny 1998, private communication). Table 1 lists chosen values for $V$ and $B-V$ in columns (10) and (11) with references noted in column (12). Similarly, spectral type is chosen from the Mermilliod \& Mermilliod (1994) compilation. For stars without suitable archive spectral type, the spectral type determined by Penprase (1992) from low-resolution spectroscopic methods is used. If possible, spectral type determined from photometric methods alone is not used because the uncertainty in such methods is several subclasses (Penprase 1992). In some cases (indicated by multiple references in Table 1), only the luminosity class is from the photometric methods of Penprase (1992). For the remaining stars, spectral type is taken from elsewhere in the literature or from the SIMBAD database. Chosen spectral types are listed in column (13) of Table 1 with references noted in column (14). In cases in which discrepant spectral types exist in the literature, the spectral type listed is the one most consistent with optical and infrared colors. We assume early-type stars with no cataloged luminosity class to be dwarfs. Stars $\mathrm{G}$ and later with no cataloged luminosity class are assumed to be giants on the basis of the small distance that would be implied if they were dwarfs. Intrinsic $(B-V)_{0}$ colors from Schmidt-Kaler (1982) are used to calculate blue visual color excess, listed in column (15) of Table 1. Uncertainty in reddening, listed in column (16), is estimated from the precision of the spectral type and photometric data sources, as well as from the discrepancy among different published measurements. Where $B-V$ is missing in column (13) of Table 1, the reddening $E_{B-V}$ is not calculated but instead is quoted from Penprase (1992), who uses intermediate-band photometry to estimate $V$ and $E_{B-V}$ directly.

Because the zero point and scale of different NIR detector systems can vary by several hundredths of a magnitude (Bessell 
TABLE 1

Photometry and Color Excess

\begin{tabular}{|c|c|c|c|c|c|c|c|c|c|c|c|c|c|c|c|c|c|c|c|}
\hline $\begin{array}{l}\text { Name } \\
\text { (1) }\end{array}$ & $\begin{array}{c}J \\
(2)\end{array}$ & $\begin{array}{l}\sigma J \\
(3)\end{array}$ & $\begin{array}{r}H \\
(4)\end{array}$ & $\begin{array}{l}\sigma H \\
(5)\end{array}$ & $\begin{array}{r}K \\
(6)\end{array}$ & $\begin{array}{l}\sigma K \\
\text { (7) }\end{array}$ & $\begin{array}{r}L \\
(8)\end{array}$ & $\begin{array}{l}\sigma L \\
(9)\end{array}$ & $\begin{array}{c}V \\
(10)\end{array}$ & $\begin{array}{c}B-V \\
(11)\end{array}$ & $\begin{array}{l}\text { Reference }^{\mathrm{a}} \\
\text { (12) }\end{array}$ & $\begin{array}{l}\text { Spectral Type } \\
\text { (13) }\end{array}$ & $\begin{array}{l}\text { Reference }^{\mathrm{b}} \\
\text { (14) }\end{array}$ & $\begin{array}{r}E_{B-V} \\
(15)\end{array}$ & $\begin{array}{c}\sigma E_{B-V} \\
(16)\end{array}$ & $\begin{array}{r}E_{V-J} \\
(17)\end{array}$ & $\begin{array}{c}E_{V-H} \\
(18)\end{array}$ & $\begin{array}{c}E_{V-K} \\
(19)\end{array}$ & $\begin{array}{c}E_{V-L} \\
(20)\end{array}$ \\
\hline HD $5931 \ldots \ldots \ldots \ldots . . .$. & 6.73 & 0.03 & 6.10 & 0.02 & 6.00 & 0.02 & 5.91 & 0.05 & 8.79 & 1.29 & 1 & K2 & $\mathrm{s}$ & 0.17 & 0.10 & 0.33 & 0.36 & 0.37 & 0.39 \\
\hline HD 6667 .................... & 8.91 & 0.02 & 8.73 & 0.02 & 8.69 & 0.03 & $\ldots$ & $\ldots$ & 9.51 & & $\mathrm{p}$ & A2 V & $\mathrm{s}, \mathrm{p}$ & 0.23 & 0.08 & 0.49 & 0.63 & 0.67 & $\ldots$ \\
\hline 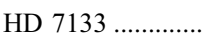 & 8.14 & 0.02 & 7.98 & 0.03 & 7.94 & 0.03 & $\ldots$ & $\ldots$ & 8.91 & 0.27 & $\mathrm{p}$ & $\mathrm{A} 3 \mathrm{~V}$ & $\mathrm{~s}, \mathrm{p}$ & 0.19 & 0.06 & 0.60 & 0.71 & 0.74 & $\ldots$ \\
\hline HD $7162 \ldots \ldots \ldots \ldots . . .$. & 6.78 & 0.02 & 6.24 & 0.02 & 6.16 & 0.03 & $\ldots$ & $\ldots$ & 8.42 & 0.91 & 1 & G5 & $\mathrm{s}$ & 0.23 & 0.10 & 0.48 & 0.64 & 0.66 & $\ldots$ \\
\hline HD $8153 \ldots \ldots \ldots \ldots . . . .$. & 6.90 & 0.06 & 6.82 & 0.06 & 6.77 & 0.06 & $\ldots$ & $\ldots$ & 7.40 & 0.29 & 1 & A0 & $\mathrm{s}$ & 0.31 & 0.10 & 0.50 & 0.57 & 0.62 & $\ldots$ \\
\hline HD $18873 \ldots \ldots \ldots . . .$. & 8.10 & 0.02 & 7.86 & 0.02 & 7.82 & 0.03 & 7.78 & 0.08 & 8.86 & 0.41 & $\mathrm{p}$ & A5 V & $\mathrm{s}, \mathrm{p}$ & 0.26 & 0.05 & 0.46 & 0.62 & 0.65 & 0.66 \\
\hline HD 19107 .............. & 4.94 & 0.02 & 4.80 & 0.02 & 4.78 & 0.03 & 4.74 & 0.05 & 5.26 & 0.21 & $\mathrm{~m}$ & A8 V & $\mathrm{m}$ & -0.04 & 0.04 & -0.11 & -0.09 & -0.10 & -0.10 \\
\hline SAO $130278 \ldots \ldots .$. & 9.18 & 0.02 & 9.11 & 0.02 & 9.07 & 0.03 & $\ldots$ & $\ldots$ & 9.45 & 0.15 & $\mathrm{p}$ & A $0 \mathrm{~V}$ & $\mathrm{~s}, \mathrm{p}$ & 0.17 & 0.05 & 0.27 & 0.33 & 0.37 & $\ldots$ \\
\hline HD 19462 .............. & 7.60 & 0.02 & 7.47 & 0.02 & 7.45 & 0.03 & 7.41 & 0.06 & 8.10 & $\ldots$ & $\mathrm{p}$ & A $0 \mathrm{~V}$ & $\mathrm{~s}, \mathrm{p}$ & 0.21 & 0.06 & 0.50 & 0.62 & 0.64 & 0.67 \\
\hline SAO $111084 \ldots \ldots .$. & 8.52 & 0.02 & 8.26 & 0.02 & 8.22 & 0.03 & $\ldots$ & $\ldots$ & 9.40 & $\ldots$ & $\mathrm{p}$ & F1 V & $\mathrm{p}$ & 0.12 & 0.06 & 0.31 & 0.40 & 0.41 & $\ldots$ \\
\hline HD $20319 \ldots \ldots \ldots . .$. & 6.14 & 0.02 & 6.08 & 0.02 & 6.09 & 0.03 & 6.08 & 0.05 & 6.16 & -0.02 & $\mathrm{~m}$ & B9 V & $\mathrm{m}$ & 0.05 & 0.04 & 0.11 & 0.18 & 0.19 & 0.19 \\
\hline HD $21613 \ldots \ldots \ldots . . . .$. & 8.39 & 0.02 & 8.19 & 0.02 & 8.16 & 0.03 & $\ldots$ & $\ldots$ & 9.08 & $\ldots$ & $\mathrm{p}$ & A7 V & $\mathrm{s}, \mathrm{p}$ & 0.16 & 0.09 & 0.31 & 0.40 & 0.41 & $\ldots$ \\
\hline HD $21777 \ldots \ldots \ldots . . .$. & 7.33 & 0.02 & 7.22 & 0.02 & 7.21 & 0.02 & $\ldots$ & $\ldots$ & 7.71 & $\ldots$ & $\mathrm{p}$ & A2 V & $\mathrm{s}, \mathrm{p}$ & 0.11 & 0.09 & 0.27 & 0.34 & 0.35 & $\ldots$ \\
\hline HD $23095 \ldots \ldots \ldots \ldots . .$. & 6.80 & 0.02 & 6.70 & 0.02 & 6.68 & 0.03 & 6.63 & 0.05 & 7.08 & $\ldots$ & $\mathrm{p}$ & A1 IV/V & $\mathrm{s}$ & 0.12 & 0.06 & 0.23 & 0.30 & 0.32 & 0.36 \\
\hline HD $23466 \ldots \ldots \ldots \ldots . . .$. & 5.55 & 0.02 & 5.59 & 0.02 & 5.63 & 0.03 & 5.70 & 0.07 & 5.35 & -0.11 & $\mathrm{~m}$ & B3 V & $\mathrm{m}$ & 0.10 & 0.04 & 0.23 & 0.26 & 0.28 & 0.25 \\
\hline HD $24003 \ldots \ldots \ldots . . .$. & 8.52 & 0.02 & 8.50 & 0.02 & 8.50 & 0.04 & $\ldots$ & $\ldots$ & 8.59 & 0.04 & $\mathrm{k}$ & B9 & $\mathrm{s}$ & 0.11 & 0.06 & 0.16 & 0.19 & 0.21 & $\ldots$ \\
\hline HD $24264 \ldots \ldots \ldots . .$. & 8.91 & 0.02 & 8.74 & 0.02 & 8.68 & 0.03 & $\ldots$ & $\ldots$ & 9.71 & 0.40 & $\mathrm{f}$ & $\mathrm{A} 3$ & $\mathrm{~s}$ & 0.32 & 0.10 & 0.63 & 0.75 & 0.80 & $\ldots$ \\
\hline HD $24263 \ldots \ldots \ldots . .$. & 5.48 & 0.02 & 5.47 & 0.02 & 5.49 & 0.03 & 5.49 & 0.05 & 5.66 & 0.06 & $\mathrm{~m}$ & B5 V & $\mathrm{m}$ & 0.23 & 0.04 & 0.54 & 0.61 & 0.64 & 0.70 \\
\hline HD $24400 \ldots \ldots \ldots \ldots . .$. & 6.66 & 0.03 & 6.41 & 0.02 & 6.38 & 0.03 & 6.36 & 0.05 & 7.48 & 0.50 & $\mathrm{k}$ & F5 & $\mathrm{s}$ & 0.06 & 0.06 & 0.00 & -0.00 & -0.01 & -0.04 \\
\hline HD $24520 \ldots \ldots \ldots . . . .$. & 8.32 & 0.02 & 8.25 & 0.02 & 8.22 & 0.03 & 8.33 & 0.06 & 8.57 & 0.14 & $\mathrm{p}$ & B9 V & $\mathrm{s}, \mathrm{p}$ & 0.21 & 0.05 & 0.34 & 0.42 & 0.47 & 0.35 \\
\hline HD $24749 \ldots \ldots \ldots . .$. & 8.50 & 0.02 & 8.25 & 0.02 & 8.19 & 0.03 & 8.11 & 0.11 & 9.46 & 0.47 & $\mathrm{~m}$ & F0 V & $\mathrm{m}, \mathrm{p}$ & 0.17 & 0.05 & 0.43 & 0.53 & 0.56 & 0.60 \\
\hline HD $24763 \ldots \ldots \ldots . . . . .$. & 6.37 & 0.02 & 5.81 & 0.03 & 5.70 & 0.03 & 5.63 & 0.05 & 8.26 & 1.10 & $\mathrm{k}$ & K0 & $\mathrm{s}$ & 0.29 & 0.10 & 0.48 & 0.55 & 0.59 & 0.59 \\
\hline HD $24819 \ldots \ldots \ldots . .$. & 7.42 & 0.02 & 7.27 & 0.02 & 7.24 & 0.03 & 7.26 & 0.05 & 8.09 & 0.30 & $\mathrm{p}$ & A2 V & $\mathrm{s}, \mathrm{p}$ & 0.25 & 0.05 & 0.56 & 0.67 & 0.70 & 0.66 \\
\hline SAO $111522 \ldots \ldots .$. & 8.52 & 0.02 & 8.28 & 0.03 & 8.22 & 0.03 & 8.23 & 0.15 & 9.69 & 0.54 & $\mathrm{p}$ & A5 V & $\mathrm{s}, \mathrm{p}$ & 0.39 & 0.05 & 0.87 & 1.03 & 1.08 & 1.04 \\
\hline HD $25093 \ldots \ldots \ldots . . . .$. & 8.38 & 0.02 & 8.18 & 0.02 & 8.14 & 0.03 & 8.17 & 0.16 & 9.24 & 0.45 & $\mathrm{p}$ & A2 V & $\mathrm{s}, \mathrm{p}$ & 0.40 & 0.05 & 0.75 & 0.91 & 0.95 & 1.04 \\
\hline HD $25450 \ldots \ldots \ldots . .$. & 8.15 & 0.03 & 7.91 & 0.03 & 7.86 & 0.04 & 7.80 & 0.08 & 8.98 & 0.41 & $\mathrm{~m}$ & F0 IV & $\mathrm{m}$ & 0.11 & 0.04 & 0.30 & 0.39 & 0.41 & 0.43 \\
\hline SAO $111548 \ldots \ldots . .$. & 8.71 & 0.02 & 8.44 & 0.02 & 8.39 & 0.03 & 8.15 & 0.06 & 9.81 & 0.55 & $\mathrm{p}$ & F5 V & $\mathrm{s}, \mathrm{p}$ & 0.11 & 0.05 & 0.28 & 0.30 & 0.31 & 0.50 \\
\hline HD $25137 \ldots \ldots \ldots . .$. & 7.03 & 0.02 & 6.98 & 0.03 & 6.97 & 0.02 & 6.99 & 0.06 & 7.29 & 0.11 & $\mathrm{p}$ & B9 V & $\mathrm{p}$ & 0.18 & 0.05 & 0.35 & 0.41 & 0.44 & 0.41 \\
\hline HD $25154 \ldots \ldots \ldots \ldots . .$. & 8.64 & 0.02 & 8.40 & 0.02 & 8.31 & 0.03 & 8.35 & 0.07 & 9.86 & 0.65 & $\mathrm{p}$ & A5 V & $\mathrm{s}, \mathrm{p}$ & 0.50 & 0.05 & 0.92 & 1.08 & 1.16 & 1.09 \\
\hline HD $25339 \ldots \ldots \ldots . . . .$. & 8.53 & 0.02 & 8.30 & 0.02 & 8.24 & 0.03 & 8.09 & 0.10 & 9.54 & 0.49 & $\mathrm{p}$ & F0 V & $\mathrm{s}, \mathrm{p}$ & 0.19 & 0.05 & 0.48 & 0.56 & 0.59 & 0.70 \\
\hline HD $25863 \ldots \ldots \ldots . . .$. & 9.39 & 0.03 & 9.24 & 0.03 & 9.20 & 0.04 & $\ldots$ & $\ldots$ & 9.92 & 0.33 & $\mathrm{p}$ & $\mathrm{A} 2 / \mathrm{A} 3 \mathrm{~V}$ & $\mathrm{~s}$ & 0.27 & 0.05 & 0.39 & 0.49 & 0.53 & $\ldots$ \\
\hline HD $25400 \ldots \ldots \ldots . .$. & 7.66 & 0.02 & 7.48 & 0.02 & 7.47 & 0.03 & 7.41 & 0.05 & 8.33 & 0.33 & $\mathrm{p}$ & A3 & $\mathrm{s}$ & 0.25 & 0.05 & 0.50 & 0.63 & 0.63 & 0.67 \\
\hline HD $25413 \ldots \ldots \ldots . .$. & 8.27 & 0.02 & 8.05 & 0.02 & 8.01 & 0.03 & 7.92 & 0.05 & 9.23 & 0.46 & $\mathrm{p}$ & B8 V & $\mathrm{s}$ & 0.57 & 0.05 & 1.12 & 1.38 & 1.46 & 1.55 \\
\hline HD $25520 \ldots \ldots \ldots . . . .$. & 4.17 & 0.03 & 3.26 & 0.04 & 3.10 & 0.04 & 2.92 & 0.06 & 7.07 & 1.68 & $\mathrm{k}$ & $\mathrm{K} 2$ & $\mathrm{~s}$ & 0.77 & 0.10 & 1.30 & 1.67 & 1.74 & 1.84 \\
\hline HD $25881 \ldots \ldots \ldots . . .$. & 8.57 & 0.02 & 8.43 & 0.02 & 8.39 & 0.03 & 8.02 & 0.08 & 9.26 & & $\mathrm{p}$ & B9 $\mathrm{V}$ & $\mathrm{p}$ & 0.34 & 0.07 & 0.78 & 0.93 & 0.99 & 1.35 \\
\hline HD $25896 \ldots \ldots \ldots . . . .$. & 6.59 & 0.02 & 6.00 & 0.02 & 5.91 & 0.02 & 5.85 & 0.05 & 8.36 & 1.03 & $\mathrm{k}$ & G5 & $\mathrm{s}$ & 0.17 & 0.05 & 0.32 & 0.45 & 0.47 & 0.45 \\
\hline HD $26434 \ldots \ldots \ldots \ldots$ & 9.61 & 0.03 & 9.50 & 0.03 & 9.46 & 0.04 & $\ldots$ & $\ldots$ & 10.05 & 0.25 & $\mathrm{p}$ & A1 V & $\mathrm{s}$ & 0.24 & 0.06 & 0.39 & 0.47 & 0.51 & $\ldots$ \\
\hline HD $26173 \ldots \ldots \ldots . . .$. & 8.55 & 0.03 & 8.42 & 0.02 & 8.36 & 0.03 & $\ldots$ & $\ldots$ & 9.01 & 0.26 & $\mathrm{k}$ & $\mathrm{A} 0$ & $\mathrm{~s}$ & 0.28 & 0.06 & 0.46 & 0.58 & 0.64 & $\ldots$ \\
\hline SAO $111632 \ldots \ldots$. & 8.58 & 0.02 & 8.33 & 0.02 & 8.25 & 0.03 & 8.26 & 0.15 & 9.84 & 0.56 & $\mathrm{p}$ & A2 V & $\mathrm{p}$ & 0.51 & 0.05 & 1.15 & 1.36 & 1.44 & 1.41 \\
\hline HD $26323 \ldots \ldots \ldots . . .$. & 7.94 & 0.02 & 7.80 & 0.02 & 7.77 & 0.03 & 7.76 & 0.08 & 8.64 & 0.32 & $\mathrm{p}$ & B9 IV & $\mathrm{s}, \mathrm{p}$ & 0.39 & 0.05 & 0.79 & 0.94 & 0.99 & 0.99 \\
\hline SAO $111655 \ldots \ldots$. & 8.46 & 0.02 & 8.25 & 0.02 & 8.15 & 0.03 & 8.14 & 0.07 & 9.72 & 0.56 & $\mathrm{p}$ & A2 V & $\mathrm{p}$ & 0.51 & 0.05 & 1.15 & 1.32 & 1.42 & 1.41 \\
\hline HD $26607 \ldots \ldots \ldots . . .$. & 6.63 & 0.03 & 5.97 & 0.04 & 5.87 & 0.04 & 5.80 & 0.05 & 8.71 & 1.23 & $\mathrm{k}$ & G5 & $\mathrm{s}$ & 0.55 & 0.10 & 0.92 & 1.20 & 1.24 & 1.25 \\
\hline HD $26691 \ldots \ldots \ldots . . .$. & 4.29 & 0.03 & 3.23 & 0.04 & 3.01 & 0.04 & 2.80 & 0.05 & 8.29 & 1.86 & $\mathrm{k}$ & K5 & $\mathrm{s}$ & 0.71 & 0.10 & 1.90 & 2.31 & 2.42 & 2.52 \\
\hline HD $26738 \ldots \ldots \ldots . . .$. & 8.70 & 0.03 & 8.51 & 0.03 & 8.48 & 0.03 & 8.42 & 0.16 & 9.52 & 0.38 & $\mathrm{k}$ & $\mathrm{A} 0$ & $\mathrm{~s}$ & 0.40 & 0.06 & 0.82 & 1.00 & 1.03 & 1.08 \\
\hline HD $27063 \ldots \ldots \ldots . . . .$. & 6.95 & 0.02 & 6.61 & 0.02 & 6.56 & 0.03 & & $\ldots$ & 8.05 & 0.67 & $\mathrm{k}$ & G0 & $\mathrm{s}$ & 0.09 & 0.10 & 0.06 & 0.07 & 0.07 & $\ldots$ \\
\hline HD $27146 \ldots \ldots \ldots . .$. & 5.48 & 0.03 & 4.89 & 0.04 & 4.82 & 0.04 & 4.70 & 0.05 & 7.22 & 1.06 & $\mathrm{k}$ & G5 & $\mathrm{s}$ & 0.38 & 0.10 & 0.58 & 0.79 & 0.80 & 0.87 \\
\hline HD $28856 \ldots \ldots \ldots \ldots . .$. & 9.01 & 0.02 & 8.95 & 0.02 & 8.93 & 0.03 & & & 9.32 & & $\mathrm{p}$ & A1 V & $\mathrm{s}$ & 0.08 & 0.06 & 0.26 & 0.29 & 0.31 & \\
\hline
\end{tabular}


TABLE 1-Continued

\begin{tabular}{|c|c|c|c|c|c|c|c|c|c|c|c|c|c|c|c|c|c|c|c|}
\hline $\begin{array}{l}\text { Name } \\
\text { (1) }\end{array}$ & $\begin{array}{c}J \\
(2)\end{array}$ & $\begin{array}{l}\sigma J \\
(3)\end{array}$ & $\begin{array}{r}H \\
(4)\end{array}$ & $\begin{array}{l}\sigma H \\
(5)\end{array}$ & $\begin{array}{r}K \\
(6)\end{array}$ & $\begin{array}{l}\sigma K \\
(7)\end{array}$ & $\begin{array}{c}L \\
(8)\end{array}$ & $\begin{array}{l}\sigma L \\
(9)\end{array}$ & $\begin{array}{c}V \\
(10)\end{array}$ & $\begin{array}{c}B-V \\
(11)\end{array}$ & $\begin{array}{l}\text { Reference }^{\mathrm{a}} \\
\text { (12) }\end{array}$ & $\begin{array}{c}\text { Spectral Type } \\
\text { (13) }\end{array}$ & $\begin{array}{c}\text { Reference }^{\mathrm{b}} \\
\text { (14) }\end{array}$ & $\begin{array}{r}E_{B-V} \\
(15)\end{array}$ & $\begin{array}{c}\sigma E_{B-V} \\
(16)\end{array}$ & $\begin{array}{l}E_{V-J} \\
(17)\end{array}$ & $\begin{array}{c}E_{V-H} \\
(18)\end{array}$ & $\begin{array}{c}E_{V-K} \\
(19)\end{array}$ & $\begin{array}{r}E_{V-L} \\
(20)\end{array}$ \\
\hline SAO $149765 \ldots \ldots \ldots . .$. & 8.76 & 0.02 & 8.37 & 0.02 & 8.27 & 0.03 & 8.13 & 0.13 & 10.47 & 0.84 & $\mathrm{p}$ & F8 V & $\mathrm{s}, \mathrm{p}$ & 0.32 & 0.05 & 0.72 & 0.78 & 0.84 & 0.93 \\
\hline HD $31900 \ldots \ldots \ldots \ldots \ldots . . .$. & 9.11 & 0.02 & 9.08 & 0.02 & 9.05 & 0.03 & $\ldots$ & $\ldots$ & 9.36 & 0.12 & $\mathrm{p}$ & $\mathrm{A} 0 \mathrm{~V}$ & $\mathrm{~s}, \mathrm{p}$ & 0.14 & 0.05 & 0.25 & 0.27 & 0.30 & $\ldots$ \\
\hline HD $34639 \ldots \ldots \ldots \ldots . . . .$. & 9.52 & 0.02 & 9.52 & 0.02 & 9.52 & 0.03 & $\ldots$ & $\ldots$ & 9.61 & 0.00 & $\mathrm{p}$ & B6 V & $\mathrm{p}$ & 0.15 & 0.05 & 0.37 & 0.41 & 0.46 & $\ldots$ \\
\hline SAO $170502 \ldots \ldots \ldots$ & 9.67 & 0.05 & 9.60 & 0.05 & 9.57 & 0.06 & & $\ldots$ & 9.95 & 0.17 & $\mathrm{p}$ & B9.5 V & $\mathrm{p}$ & 0.22 & 0.05 & 0.33 & 0.40 & 0.43 & \\
\hline HD $55718 \ldots \ldots \ldots \ldots \ldots$ & 6.24 & 0.03 & 6.29 & 0.02 & 6.34 & 0.03 & 6.38 & 0.06 & 5.94 & -0.15 & $\mathrm{~m}$ & B4 V & $\mathrm{m}$ & 0.04 & 0.05 & 0.09 & 0.11 & 0.11 & 0.13 \\
\hline HD $56073 \ldots \ldots \ldots \ldots . . .$. & 8.02 & 0.02 & 7.93 & 0.02 & 7.91 & 0.03 & 7.94 & 0.06 & 8.37 & 0.14 & $\mathrm{p}$ & A1 V & $\mathrm{s}$ & 0.13 & 0.05 & 0.30 & 0.36 & 0.38 & 0.34 \\
\hline 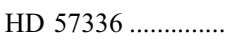 & 7.31 & 0.02 & 7.20 & 0.02 & 7.17 & 0.03 & 7.15 & 0.05 & 7.82 & 0.17 & $\mathrm{k}$ & A0 IV & $\mathrm{s}$ & 0.19 & 0.05 & 0.51 & 0.61 & 0.64 & 0.65 \\
\hline HD $59421 \ldots \ldots \ldots \ldots \ldots . .$. & 8.00 & 0.02 & 7.90 & 0.02 & 7.87 & 0.03 & 7.85 & 0.06 & 8.63 & 0.22 & $\mathrm{k}$ & B8/B9 IV/V & $\mathrm{s}$ & 0.31 & 0.05 & 0.76 & 0.88 & 0.94 & 0.96 \\
\hline HD $64033 \ldots \ldots \ldots \ldots \ldots . .$. & 8.30 & 0.02 & 8.12 & 0.02 & 8.09 & 0.03 & 8.06 & 0.05 & 8.98 & 0.36 & $\mathrm{k}$ & F2 V & $\mathrm{s}$ & 0.01 & 0.05 & 0.07 & 0.06 & 0.06 & 0.05 \\
\hline 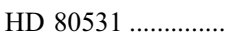 & 8.16 & 0.02 & 8.06 & 0.03 & 8.04 & 0.04 & 8.07 & 0.09 & 8.55 & $\ldots$ & $\mathrm{p}$ & A3 V & $\mathrm{s}$ & 0.13 & 0.06 & 0.22 & 0.27 & 0.28 & 0.23 \\
\hline HD $80840 \ldots \ldots \ldots \ldots \ldots . .$. & 9.37 & 0.02 & 9.28 & 0.03 & 9.23 & 0.03 & $\ldots$ & $\ldots$ & 10.04 & 0.31 & $\mathrm{k}$ & $\mathrm{A} 0 \mathrm{~V}$ & $\mathrm{~s}$ & 0.33 & 0.05 & 0.67 & 0.75 & 0.80 & $\ldots$ \\
\hline 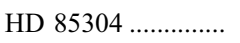 & 8.81 & 0.02 & 8.71 & 0.02 & 8.69 & 0.03 & $\ldots$ & $\ldots$ & 9.14 & 0.17 & $\mathrm{p}$ & $\mathrm{A} 2 \mathrm{~V}$ & $\mathrm{~s}, \mathrm{p}$ & 0.12 & 0.05 & 0.22 & 0.28 & 0.30 & . \\
\hline HD $94434 \ldots \ldots \ldots . . . .$. & 7.76 & 0.02 & 7.53 & 0.03 & 7.44 & 0.03 & 7.39 & 0.05 & 8.95 & 0.51 & $\mathrm{p}$ & A2 V & $\mathrm{s}$ & 0.46 & 0.05 & 1.08 & 1.27 & 1.36 & 1.39 \\
\hline 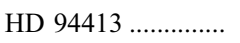 & 8.84 & 0.03 & 8.67 & 0.02 & 8.60 & 0.03 & $\ldots$ & $\ldots$ & 9.73 & 0.42 & $\mathrm{p}$ & A2 IV/V & $\mathrm{s}$ & 0.37 & 0.05 & 0.78 & 0.91 & 0.98 & $\ldots$ \\
\hline HD $94473 \ldots \ldots \ldots \ldots . . . .$. & 7.63 & 0.02 & 7.64 & 0.02 & 7.66 & 0.03 & $\ldots$ & $\ldots$ & 7.51 & -0.06 & $\mathrm{p}$ & B5 III & $\mathrm{s}$ & 0.11 & 0.05 & 0.18 & 0.21 & 0.25 & $\ldots$ \\
\hline HD 96237 ................. & 9.02 & 0.02 & 8.94 & 0.02 & 8.92 & 0.03 & $\ldots$ & $\ldots$ & 9.51 & 0.39 & $\mathrm{p}$ & $\mathrm{A} 2 \mathrm{~V}$ & $\mathrm{~s}, \mathrm{p}$ & 0.34 & 0.15 & 0.38 & 0.42 & 0.44 & $\ldots$ \\
\hline SAO $179675 \ldots \ldots \ldots . .$. & 10.27 & 0.02 & 10.11 & 0.02 & 10.07 & 0.04 & $\ldots$ & $\ldots$ & 10.95 & 0.32 & $\mathrm{p}$ & A5 V & $\mathrm{s}, \mathrm{p}$ & 0.17 & 0.05 & 0.38 & 0.46 & 0.49 & $\ldots$ \\
\hline HD $98837 \ldots \ldots \ldots \ldots . . .$. & 7.68 & 0.02 & 7.50 & 0.03 & 7.44 & 0.04 & 7.40 & 0.05 & 8.54 & 0.40 & $\mathrm{p}$ & A3 V & $\mathrm{s}$ & 0.32 & 0.05 & 0.68 & 0.81 & 0.87 & 0.89 \\
\hline HD $99786 \ldots \ldots \ldots \ldots . . .$. & 8.55 & 0.02 & 8.47 & 0.02 & 8.46 & 0.04 & $\ldots$ & $\ldots$ & 9.04 & 0.18 & $\mathrm{p}$ & B9 IV/V & $\mathrm{s}$ & 0.25 & 0.05 & 0.58 & 0.67 & 0.70 & . \\
\hline HD $100359 \ldots \ldots \ldots . . .$. & 6.35 & 0.02 & 6.26 & 0.02 & 6.22 & 0.03 & 6.21 & 0.05 & 6.88 & 0.26 & $\mathrm{~m}$ & B7 IV & $\mathrm{s}$ & 0.39 & 0.04 & 0.72 & 0.85 & 0.92 & 0.98 \\
\hline HD $106631 \ldots \ldots \ldots \ldots . .$. & 8.65 & 0.02 & 8.50 & 0.02 & 8.48 & 0.03 & 8.47 & 0.05 & 9.26 & 0.36 & $\mathrm{p}$ & A1 V & $\mathrm{s}, \mathrm{p}$ & 0.35 & 0.05 & 0.55 & 0.68 & 0.70 & 0.70 \\
\hline HD 125679 ............... & 8.49 & 0.02 & 8.44 & 0.02 & 8.42 & 0.03 & 8.35 & 0.08 & 8.84 & 0.20 & $\mathrm{p}$ & $\mathrm{A} 1 / \mathrm{A} 2 \mathrm{~V}$ & $\mathrm{~s}$ & 0.15 & 0.08 & 0.27 & 0.29 & 0.30 & 0.36 \\
\hline HD $139515 \ldots \ldots \ldots \ldots . .$. & 7.48 & 0.02 & 7.26 & 0.02 & 7.20 & 0.03 & $\ldots$ & $\ldots$ & 8.37 & 0.41 & $\mathrm{p}$ & A5 V & $\mathrm{s}, \mathrm{p}$ & 0.26 & 0.05 & 0.59 & 0.73 & 0.78 & $\ldots$ \\
\hline HD $140536 \ldots \ldots \ldots \ldots . . .$. & 8.17 & 0.02 & 8.13 & 0.02 & 8.12 & 0.03 & $\ldots$ & $\ldots$ & 8.33 & 0.07 & $\mathrm{p}$ & $\mathrm{A} 0 \mathrm{~V}$ & $\mathrm{~s}, \mathrm{p}$ & 0.10 & 0.05 & 0.16 & 0.19 & 0.20 & $\ldots$ \\
\hline HD $141423 \ldots \ldots \ldots \ldots . .$. & 7.27 & 0.02 & 7.05 & 0.02 & 6.99 & 0.03 & 6.96 & 0.05 & 8.23 & 0.45 & $\mathrm{p}$ & $\mathrm{A} 0 \mathrm{~V}$ & $\mathrm{~s}, \mathrm{p}$ & 0.47 & 0.05 & 0.96 & 1.17 & 1.23 & 1.25 \\
\hline HD 141569 .............. & 6.88 & 0.02 & 6.82 & 0.02 & 6.81 & 0.03 & 6.70 & 0.05 & 7.18 & 0.08 & $\mathrm{p}$ & B9 $\mathrm{V}$ & $\mathrm{m}, \mathrm{p}$ & 0.15 & 0.05 & 0.39 & 0.46 & 0.49 & 0.59 \\
\hline HD $141657 \ldots \ldots \ldots \ldots . .$. & 7.25 & 0.02 & 6.95 & 0.02 & 6.87 & 0.03 & 6.81 & 0.05 & 8.51 & 0.69 & $\mathrm{p}$ & F2 V & $\mathrm{s}, \mathrm{p}$ & 0.34 & 0.05 & 0.65 & 0.76 & 0.81 & 0.83 \\
\hline HD $142554 \ldots \ldots \ldots \ldots . .$. & 9.19 & 0.02 & 9.13 & 0.02 & 9.12 & 0.03 & $\ldots$ & $\ldots$ & 9.79 & 0.19 & $\mathrm{p}$ & $\mathrm{A} 0 \mathrm{~V}$ & $\mathrm{~s}, \mathrm{p}$ & 0.21 & 0.05 & 0.60 & 0.65 & 0.66 & $\ldots$ \\
\hline HD $142863 \ldots \ldots \ldots \ldots$. & 8.08 & 0.02 & 7.90 & 0.02 & 7.83 & 0.03 & 7.80 & 0.10 & 8.97 & 0.40 & $\mathrm{p}$ & $\mathrm{A} 0 \mathrm{~V}$ & $\mathrm{~s}, \mathrm{p}$ & 0.42 & 0.05 & 0.89 & 1.06 & 1.13 & 1.15 \\
\hline HD $142930 \ldots \ldots \ldots . .$. & 6.85 & 0.02 & 6.76 & 0.02 & 6.74 & 0.03 & 6.71 & 0.05 & 7.29 & 0.21 & $\mathrm{p}$ & $\mathrm{A} 0 \mathrm{~V}$ & $\mathrm{~s}, \mathrm{p}$ & 0.23 & 0.05 & 0.44 & 0.52 & 0.54 & 0.56 \\
\hline HD $143294 \ldots \ldots \ldots \ldots .$. & 8.63 & 0.02 & 8.43 & 0.02 & 8.38 & 0.03 & $\ldots$ & $\ldots$ & 9.41 & 0.35 & $\mathrm{p}$ & $\mathrm{A} 2 \mathrm{~V}$ & $\mathrm{~s}, \mathrm{p}$ & 0.30 & 0.05 & 0.67 & 0.83 & 0.88 & $\ldots$ \\
\hline HD $145631 \ldots \ldots \ldots . .$. & 7.10 & 0.03 & 7.01 & 0.03 & 6.98 & 0.04 & 6.89 & 0.06 & 7.59 & 0.15 & $\mathrm{~m}$ & B9 V & $\mathrm{m}$ & 0.22 & 0.04 & 0.58 & 0.68 & 0.73 & 0.81 \\
\hline HD $146931 \ldots \ldots \ldots . . .$. & 7.15 & 0.02 & 7.02 & 0.02 & 6.97 & 0.03 & 6.97 & 0.05 & 7.81 & 0.27 & $\mathrm{p}$ & $\mathrm{A} 0 \mathrm{~V}$ & $\mathrm{~s}, \mathrm{p}$ & 0.29 & 0.05 & 0.66 & 0.78 & 0.83 & 0.82 \\
\hline HD $147010 \ldots \ldots \ldots \ldots$ & 6.82 & 0.03 & 6.73 & 0.03 & 6.72 & 0.04 & 6.70 & 0.06 & 7.40 & 0.15 & $\mathrm{~m}$ & B9 II/III & $\mathrm{s}$ & 0.22 & 0.04 & 0.51 & 0.59 & 0.59 & 0.60 \\
\hline HD 153212 ............... & 8.02 & 0.02 & 7.78 & 0.02 & 7.70 & 0.03 & 7.65 & 0.05 & 9.12 & 0.56 & $\mathrm{p}$ & A3 V & $\mathrm{s}, \mathrm{p}$ & 0.48 & 0.08 & 0.93 & 1.12 & 1.19 & 1.22 \\
\hline HD $153605 \ldots \ldots \ldots \ldots . . .$. & 7.49 & 0.02 & 7.31 & 0.02 & 7.24 & 0.02 & 7.15 & 0.05 & 8.53 & 0.48 & $\mathrm{p}$ & A3 V & $\mathrm{s}, \mathrm{p}$ & 0.40 & 0.08 & 0.86 & 1.00 & 1.06 & 1.13 \\
\hline HD $156225 \ldots \ldots \ldots \ldots \ldots$ & 8.37 & 0.02 & 8.21 & 0.02 & 8.17 & 0.03 & 8.26 & 0.09 & 8.97 & 0.26 & $\mathrm{p}$ & A2 V & $\mathrm{s}, \mathrm{p}$ & 0.21 & 0.05 & 0.49 & 0.61 & 0.65 & 0.54 \\
\hline HD $156597 \ldots \ldots \ldots \ldots . .$. & 8.32 & 0.02 & 8.02 & 0.02 & 7.90 & 0.03 & 7.91 & 0.06 & 9.88 & 0.65 & $\mathrm{p}$ & A $0 \mathrm{~V}$ & $\mathrm{~s}, \mathrm{p}$ & 0.67 & 0.05 & 1.56 & 1.85 & 1.97 & 1.95 \\
\hline HD $156822 \ldots \ldots \ldots \ldots . .$. & 7.52 & 0.02 & 7.47 & 0.02 & 7.47 & 0.03 & 7.49 & 0.07 & 7.70 & 0.04 & $\mathrm{p}$ & A0 $\mathrm{V}$ & $\mathrm{s}, \mathrm{p}$ & 0.06 & 0.05 & 0.18 & 0.22 & 0.22 & 0.19 \\
\hline HD $156912 \ldots \ldots \ldots . . .$. & 9.71 & 0.09 & 9.47 & 0.04 & 9.42 & 0.05 & $\ldots$ & $\ldots$ & 9.55 & 0.20 & $\mathrm{p}$ & A6 V & $\mathrm{p}$ & 0.03 & 0.05 & -0.50 & -0.35 & -0.32 & \\
\hline HD $157280 \ldots \ldots \ldots . .$. & 7.41 & 0.02 & 7.10 & 0.02 & 6.98 & 0.03 & 6.89 & 0.05 & 9.08 & 0.69 & $\mathrm{p}$ & $\mathrm{A} 2 \mathrm{~V}$ & $\mathrm{~s}, \mathrm{p}$ & 0.64 & 0.05 & 1.56 & 1.83 & 1.95 & 2.02 \\
\hline HD $157333 \ldots \ldots \ldots . . .$. & 6.89 & 0.02 & 6.61 & 0.02 & 6.49 & 0.03 & 6.39 & 0.05 & 8.60 & 0.74 & $\mathrm{p}$ & $\mathrm{A} 0 \mathrm{~V}$ & $\mathrm{~s}, \mathrm{p}$ & 0.76 & 0.05 & 1.71 & 1.98 & 2.10 & 2.19 \\
\hline HD $157512 \ldots \ldots \ldots . . .$. & 7.92 & 0.02 & 7.77 & 0.02 & 7.73 & 0.03 & 7.60 & 0.07 & 8.39 & 0.22 & $\mathrm{p}$ & A4 V & $\mathrm{s}$ & 0.11 & 0.05 & 0.23 & 0.32 & 0.35 & 0.45 \\
\hline HD $187558 \ldots \ldots \ldots . . .$. & 6.76 & 0.03 & 6.00 & 0.02 & 5.89 & 0.03 & 5.76 & 0.05 & 8.90 & 1.40 & $\mathrm{~s}$ & K0/K1 IV & $\mathrm{s}$ & 0.46 & 0.10 & 0.68 & 0.94 & 0.97 & 1.03 \\
\hline HD 187869 ................ & 8.73 & 0.02 & 8.70 & 0.02 & 8.70 & 0.03 & $\ldots$ & $\ldots$ & 8.94 & 0.07 & $\mathrm{p}$ & B9 IV/V & $\mathrm{s}$ & 0.14 & 0.08 & 0.30 & 0.34 & 0.36 & \\
\hline HD $188220 \ldots \ldots \ldots \ldots . .$. & 7.30 & 0.02 & 7.17 & 0.02 & 7.13 & 0.03 & 7.16 & 0.05 & 8.04 & 0.35 & $\mathrm{p}$ & A0 V & $\mathrm{s}$ & 0.37 & 0.08 & 0.74 & 0.86 & 0.90 & 0.86 \\
\hline HD $188350 \ldots \ldots \ldots \ldots . .$. & 5.30 & 0.02 & 5.24 & 0.02 & 5.22 & 0.03 & 5.22 & 0.05 & 5.63 & 0.10 & $\mathrm{~m}$ & A0 III & $\mathrm{s}$ & 0.13 & 0.04 & 0.33 & 0.38 & 0.40 & 0.39 \\
\hline HD 189115.............. & 6.73 & 0.03 & 5.82 & 0.02 & 5.66 & 0.03 & 5.51 & 0.06 & 9.40 & 1.40 & $\mathrm{~s}$ & K1/K2 III & $\mathrm{s}$ & 0.28 & 0.10 & 0.94 & 1.25 & 1.32 & 1.40 \\
\hline
\end{tabular}


TABLE 1-Continued

\begin{tabular}{|c|c|c|c|c|c|c|c|c|c|c|c|c|c|c|c|c|c|c|c|}
\hline $\begin{array}{l}\text { Name } \\
\text { (1) }\end{array}$ & $\begin{array}{c}J \\
(2)\end{array}$ & $\begin{array}{l}\sigma J \\
(3)\end{array}$ & $\begin{array}{r}H \\
(4)\end{array}$ & $\begin{array}{l}\sigma H \\
(5)\end{array}$ & $\begin{array}{r}K \\
(6)\end{array}$ & $\begin{array}{l}\sigma K \\
(7)\end{array}$ & $\begin{array}{c}L \\
(8)\end{array}$ & $\begin{array}{l}\sigma L \\
(9)\end{array}$ & $\begin{array}{c}V \\
(10)\end{array}$ & $\begin{array}{c}B-V \\
(11)\end{array}$ & $\begin{array}{l}\text { Reference }^{\mathrm{a}} \\
\text { (12) }\end{array}$ & $\begin{array}{c}\text { Spectral Type } \\
\text { (13) }\end{array}$ & $\begin{array}{l}\text { Reference }^{\mathrm{b}} \\
\text { (14) }\end{array}$ & $\begin{array}{c}E_{B-V} \\
(15)\end{array}$ & $\begin{array}{c}\sigma E_{B-V} \\
(16)\end{array}$ & $\begin{array}{l}E_{V-J} \\
(17)\end{array}$ & $\begin{array}{c}E_{V-H} \\
(18)\end{array}$ & $\begin{array}{c}E_{V-K} \\
(19)\end{array}$ & $\begin{array}{l}E_{V-L} \\
(20)\end{array}$ \\
\hline HD $192435 \ldots \ldots \ldots \ldots \ldots$ & 7.71 & 0.02 & 7.39 & 0.02 & 7.31 & 0.03 & $\ldots$ & $\ldots$ & 9.15 & 0.60 & $\mathrm{p}$ & F2 V & $\mathrm{s}$ & 0.25 & 0.06 & 0.83 & 0.96 & 1.01 & $\ldots$ \\
\hline HD $198772 \ldots \ldots \ldots \ldots . . .$. & 9.48 & 0.02 & 9.42 & 0.02 & 9.42 & 0.03 & $\ldots$ & $\ldots$ & 9.68 & 0.07 & $\mathrm{p}$ & $\mathrm{A} 0 \mathrm{~V}$ & $\mathrm{~s}, \mathrm{p}$ & 0.10 & 0.08 & 0.20 & 0.25 & 0.25 & $\ldots$ \\
\hline HD $199469 \ldots \ldots \ldots \ldots . .$. & 7.50 & 0.02 & 7.51 & 0.02 & 7.52 & 0.03 & $\ldots$ & $\ldots$ & 7.51 & 0.01 & $\mathrm{p}$ & $\mathrm{A} 0 \mathrm{~V}$ & $\mathrm{~s}, \mathrm{p}$ & 0.03 & 0.08 & 0.01 & -0.01 & -0.02 & \\
\hline HD $201379 \ldots \ldots \ldots \ldots . . .$. & 8.21 & 0.02 & 8.18 & 0.02 & 8.18 & 0.03 & $\ldots$ & $\ldots$ & 8.39 & 0.08 & $\mathrm{p}$ & $\mathrm{A} 0 \mathrm{~V}$ & $\mathrm{~s}, \mathrm{p}$ & 0.10 & 0.08 & 0.18 & 0.20 & 0.20 & $\ldots$ \\
\hline HD $203532^{\mathrm{c}} \ldots \ldots \ldots \ldots$ & 6.07 & 0.03 & 6.04 & 0.03 & 6.05 & 0.04 & 6.10 & 0.05 & 6.37 & 0.12 & $\mathrm{~m}$ & B3 IV/V & $\mathrm{m}$ & 0.32 & 0.04 & 0.69 & 0.78 & 0.81 & 0.80 \\
\hline HD $205037 \ldots \ldots \ldots \ldots . . .$. & 5.44 & 0.04 & 4.63 & 0.04 & 4.51 & 0.04 & $\ldots$ & $\ldots$ & 7.80 & 1.40 & $\mathrm{~s}$ & K1/K2 III & $\mathrm{s}$ & 0.28 & 0.10 & 0.63 & 0.84 & 0.87 & \\
\hline HD $207115 \ldots \ldots \ldots \ldots$ & 4.85 & 0.04 & 3.96 & 0.05 & 3.77 & 0.06 & $\ldots$ & $\ldots$ & 7.90 & 1.60 & $\mathrm{~s}$ & K3/K4 III & $\mathrm{s}$ & 0.27 & 0.10 & 0.59 & 0.74 & 0.81 & $\ldots$ \\
\hline HD $210121^{\mathrm{d}} \ldots \ldots \ldots \ldots \ldots$ & 7.42 & 0.02 & 7.41 & 0.02 & 7.43 & 0.03 & 7.42 & 0.05 & 7.67 & 0.20 & $\mathrm{w}$ & B3 V & $\mathrm{w}$ & 0.40 & 0.05 & 0.68 & 0.76 & 0.80 & 0.86 \\
\hline HD $216039 \ldots \ldots \ldots \ldots . . .$. & 7.69 & 0.02 & 7.53 & 0.02 & 7.51 & 0.03 & 7.50 & 0.11 & 8.18 & 0.34 & $\mathrm{p}$ & A4 V & $\mathrm{p}$ & 0.23 & 0.05 & 0.38 & 0.50 & 0.52 & 0.51 \\
\hline HD $216308 \ldots \ldots \ldots \ldots . . .$. & 6.81 & 0.02 & 6.76 & 0.02 & 6.76 & 0.03 & 6.71 & 0.05 & 7.00 & 0.11 & $\mathrm{p}$ & B9.5 V & $\mathrm{p}$ & 0.13 & 0.05 & 0.24 & 0.29 & 0.29 & 0.34 \\
\hline
\end{tabular}

NoTE.-Table 1 is also available in machine-readable form in the electronic edition of the Astrophysical Journal.

a Optical photometry references (cols. [10] and [11]).

Spectral type references (col. [13]).

c NIR photometry from Whittet \& van Breda (1980)

d NIR photometry from Larson et al. (1996).

References._-(p) Penprase 1992; (s) Simbad database; (m) Mermilliod \& Mermilliod 1994; (k) D. Kilkenny 2004, private communication; (1) this paper; (w) Welty \& Fowler 1992 ; (f) Franco 1988. 
$\&$ Brett 1988), care should be used in selecting intrinsic infrared colors to ensure that infrared color excess calculations are accurate. Glass (1997) presents intrinsic colors for the Carter (1990) system, noting that the colors are equivalent to the Bessell \& Brett (1988) intrinsic $(J-H)_{0},(H-K)_{0}$, and $(K-L)_{0}$ colors with a small linear correction based on the zero points at A0 V stars. The Bessell \& Brett (1988) curves adopted the previous SAAO Glass (1974) system as their basis, which had a $J$ filter different from the SAAO Carter (1990) system and which used an arbitrary estimate for the $H$ zero point. The two SAAO systems are similar despite these differences, so it is not surprising that the resulting curves of Glass (1997) and Bessell \& Brett (1988) are nearly equivalent. We adopt the Bessell \& Brett infrared colors with the Glass (1997) correction for stars A0 V and later. A small correction is applied to the $(V-K)_{0}$ intrinsic colors of Bessell $\&$ Brett using the Glass (1997) observations of stars with spectral types near A0 V. The intrinsic colors with respect to the reference band, $V$, are calculated with $(V-K)_{0}$ and the NIR colors. For stars from B8 V to B9.5 V, the earliest spectral types considered by Bessell \& Brett, we use the average interpolated $(V-K)_{0}$ colors for each spectral type and the Bessell \& Brett infrared colors with the Glass (1997) correction. Because the Bessell \& Brett colors do not extend earlier than B8, all earlier intrinsic colors are directly from the observations of Glass (1997). For the small number of giant-class stars in our program, intrinsic colors from the Glass (1997) observations are again used. Since the photometry of HD 203532 (Whittet \& van Breda 1980) was obtained on the older SAAO system, colors from Wegner (1994) that are consistent with the older SAAO system are used for this star. The corrections to the Bessell \& Brett system are on the order of a few hundredths of a magnitude, but inappropriate intrinsic colors can produce spurious results in color excess calculations, especially in low-extinction lines of sight (see $\S 3.2 .1$ ). Different sets of intrinsic colors, and variations on the correction procedure used to match those colors to the SAAO Carter (1990) system, have been tested with photometry of stars in our program to ensure compatibility and continuity across the entire range of spectral type in our project. Color excess between the NIR passbands and the visual are calculated with this system and are listed in columns (17)-(20) of Table 1.

We have compared SAAO photometry of program stars to the recently released $2 \mathrm{MASS}$ data. Because the 2MASS $K_{S}$ filter excludes some of the long-wavelength transmission of the Johnson $K$ filter used at SAAO, a color transformation is required to convert the data or intrinsic colors to a common system. For stars in our program, the offset $\left(K_{\mathrm{SAAO}}-K_{S}\right)$ has an average value of $-0.02 \pm 0.03$ and a range from -0.11 to +0.05 , consistent with a comparison of $2 \mathrm{MASS}$ and SAAO standards (2MASS Explanatory Supplement; Cutri et al. 2003). The color transformation observed for stars in our program,

$\left(J-K_{s}\right)_{2 \mathrm{MASS}}-(J-K)_{\mathrm{SAAO}}=-0.043(J-K)_{\mathrm{SAAO}}-0.016$,

is consistent with the standard color transform, $\left(J-K_{S}\right)_{2 \mathrm{MASS}}-$ $(J-K)_{\mathrm{SAAO}}=(-0.056 \pm 0.012)(J-K)_{\mathrm{SAAO}}+(-0.005 \pm$ 0.006) (Cutri et al. 2003). In these comparisons, we have omitted the six program stars that do not have the highest quality 2MASS photometry. In addition, we omit the star HD 156912, which for unknown reasons is significantly brighter in 2MASS than at SAAO, 0.43 mag brighter at $H$ and $K$ and 0.66 mag brighter at $J$. We discuss using 2MASS to extend our study of the highlatitude ISM in $\S 4$.

\section{HIGH-LATITUDE EXTINCTION}

\subsection{Near-Infrared Extinction Curve}

Absolute extinction toward a star generally cannot be calculated without a priori knowledge of the distance to the star. Instead, it is usually more practical to measure color excess with respect to a reference passband, traditionally chosen to be $V$. Color excess is differential extinction,

$$
E_{\lambda-V}=A_{\lambda}-A_{V},
$$

and when calculated between two adjacent passbands, indicates the slope of the broadband extinction curve in that range. Assuming that there is no gray extinction, extinction in the reference passband can be determined by extrapolating the infrared color excess to its long wavelength limit,

$$
A_{V}=-\left[E_{\lambda-V}\right]_{\lambda \rightarrow \infty}
$$

To determine the absolute extinction curve, $A_{\lambda}$, from color excess, extinction in the reference passband must be known. Alternatively, extinction in the reference passband can be inferred from color excess if the shape of the normalized extinction curve is known.

In the NIR, the color excess curve can be reliably extrapolated beyond the observed wavelengths with a model for longwavelength extinction such as a power law,

$$
\frac{A_{\lambda}}{A_{V}}=e \lambda^{-\alpha}
$$

For stars in our program with data in all four NIR passbands, we fitted a general offset power-law model to color excess,

$$
E_{\lambda-V}=A_{V}\left(\frac{A_{\lambda}}{A_{V}}-1\right)=A_{V}\left(e \lambda^{-\alpha}-1\right)
$$

using a downhill simplex method (AMOEBA) to minimize the sum of absolute difference between the model and data weighted by the photometric error in each point (Press et al. 1994). The commonly used mean square deviation $\chi^{2}$ minimization assumes that the errors in measurement have a Gaussian distribution, which is not likely to be true for color excess. The source of errors in color excess, such as error in intrinsic color due to error in spectral type, are nonlinear. Minimizing the mean absolute deviation assumes a two-sided exponential error distribution that has larger tails than a Gaussian and thus is a method that is less sensitive to outlier points than $\chi^{2}$ minimization.

Best-fit parameters determined by the fitting procedure are the long-wavelength limit $\left(A_{V}\right)$, scale $(e)$, and power-law index $(\alpha)$. The starting point for each three-parameter fit is $A_{V}=$ $1 \mathrm{mag}$ and the average curve shown by Martin \& Whittet (1990) to be valid over a wide range of environmental conditions in the solar neighborhood,

$$
\left\langle\frac{A_{\lambda}}{A_{V}}\right\rangle=(0.40 \pm 0.05) \lambda^{-1.85 \pm 0.10} .
$$

Of the 68 stars with NIR color excess in all four passbands, 64 are adequately fitted by a single offset power law, and $A_{V}$ for these stars is listed in column (2) of Table 2. Three of the four lines of sight not adequately fitted with a single offset power law have very low reddening and thus are subject to high relative uncertainty in color excess. It is also possible that the spectral 
TABLE 2

Extinction Curve Parameters

\begin{tabular}{|c|c|c|c|c|c|}
\hline \multirow[b]{2}{*}{$\begin{array}{l}\text { NAME } \\
(1)\end{array}$} & \multicolumn{3}{|c|}{$A_{V}$} & \multirow[b]{2}{*}{$\begin{array}{l}R_{V} \\
\text { (5) }\end{array}$} & \multirow[b]{2}{*}{$\begin{array}{c}\sigma R_{V} \\
\text { (6) }\end{array}$} \\
\hline & $\begin{array}{l}\text { Free Fit } \\
\text { (2) }\end{array}$ & $\begin{array}{l}\text { High-Latitude Fit } \\
\text { (3) }\end{array}$ & $\begin{array}{c}1.1 E_{V-K} \\
\text { (4) }\end{array}$ & & \\
\hline HD $5931 \ldots \ldots \ldots . . . .$. & 0.41 & 0.42 & 0.41 & 2.41 & 1.03 \\
\hline HD $6667 \ldots \ldots \ldots \ldots . . .$. & $\ldots$ & 0.72 & 0.73 & 3.13 & 0.81 \\
\hline HD $7133 \ldots \ldots \ldots . . . . .$. & $\ldots$ & 0.80 & 0.81 & 4.21 & 0.98 \\
\hline HD $7162 \ldots \ldots \ldots . . . . .$. & $\ldots$ & 0.30 & 0.31 & $\mathrm{a}$ & a \\
\hline HD $8153 \ldots \ldots \ldots \ldots$ & $\ldots$ & 0.66 & 0.68 & 2.14 & 0.55 \\
\hline HD $18873 \ldots \ldots \ldots$ & 0.71 & 0.70 & 0.71 & 2.74 & 0.41 \\
\hline HD $19107 \ldots \ldots \ldots . . . .$. & $\mathrm{b}$ & b & c & a & a \\
\hline SAO $130278 \ldots \ldots .$. & $\ldots$ & 0.38 & 0.40 & 2.23 & 0.56 \\
\hline HD $19462 \ldots \ldots \ldots . .$. & 0.69 & 0.69 & 0.70 & 3.28 & 0.71 \\
\hline SAO $111084 \ldots \ldots .$. & $\ldots$ & 0.44 & 0.45 & 3.65 & 1.38 \\
\hline HD $20319 \ldots \ldots \ldots . . .$. & 0.21 & 0.21 & 0.21 & a & $\mathrm{a}$ \\
\hline HD $21613 \ldots \ldots \ldots . . .$. & $\ldots$ & 0.44 & 0.45 & 2.73 & 1.15 \\
\hline HD $21777 \ldots \ldots \ldots . . .$. & $\ldots$ & 0.37 & 0.38 & 3.39 & 2.04 \\
\hline HD $23095 \ldots \ldots \ldots . . .$. & 0.38 & 0.34 & 0.35 & 3.14 & 1.10 \\
\hline HD $23466 \ldots \ldots \ldots . .$. & 0.30 & 0.31 & 0.30 & 3.04 & 0.97 \\
\hline HD $24003 \ldots \ldots \ldots . . .$. & $\ldots$ & 0.22 & 0.23 & 2.02 & 0.95 \\
\hline HD $24264 \ldots \ldots \ldots . .$. & $\ldots$ & 0.86 & 0.88 & 2.67 & 0.62 \\
\hline HD $24263 \ldots \ldots \ldots . .$. & 0.67 & 0.70 & 0.70 & 2.93 & 0.42 \\
\hline HD $24400 \ldots \ldots \ldots . . .$. & $\mathrm{b}$ & $\mathrm{b}$ & c & a & a \\
\hline HD $24520 \ldots \ldots \ldots . .$. & 0.51 & 0.49 & 0.52 & 2.43 & 0.47 \\
\hline HD $24749 \ldots \ldots \ldots . . .$. & 0.63 & 0.60 & 0.61 & 3.72 & 0.80 \\
\hline HD $24763 \ldots \ldots \ldots . . .$. & $\mathrm{b}$ & $\mathrm{b}$ & 0.26 & 2.55 & 1.86 \\
\hline HD $24819 \ldots \ldots \ldots . . .$. & 0.76 & 0.75 & 0.77 & 3.03 & 0.47 \\
\hline SAO $111522 \ldots \ldots .$. & 1.16 & 1.17 & 1.19 & 2.96 & 0.30 \\
\hline HD $25093 \ldots \ldots \ldots . . .$. & 1.08 & 1.02 & 1.04 & 2.69 & 0.26 \\
\hline HD $25450 \ldots \ldots \ldots . .$. & 0.50 & 0.44 & 0.45 & 4.51 & 1.16 \\
\hline SAO $111548 \ldots \ldots .$. & 0.31 & 0.25 & 0.34 & 2.85 & 1.07 \\
\hline HD $25137 \ldots \ldots \ldots . .$. & 0.47 & 0.44 & 0.49 & 2.61 & 0.57 \\
\hline HD $25154 \ldots \ldots \ldots . . .$. & 1.25 & 1.24 & 1.27 & 2.51 & 0.20 \\
\hline HD $25339 \ldots \ldots \ldots . . .$. & 0.65 & 0.64 & 0.65 & 3.40 & 0.68 \\
\hline HD $25863 \ldots \ldots \ldots . . .$. & $\ldots$ & 0.57 & 0.58 & 2.14 & 0.36 \\
\hline HD $25400 \ldots \ldots \ldots . .$. & 0.69 & 0.68 & 0.69 & 2.75 & 0.43 \\
\hline HD $25413 \ldots \ldots \ldots . .$. & 1.62 & 1.58 & 1.60 & 2.84 & 0.19 \\
\hline HD $25520 \ldots \ldots \ldots . . .$. & 1.73 & 1.67 & 1.71 & 3.09 & 0.40 \\
\hline HD $25881 \ldots \ldots \ldots . . .$. & 1.13 & 1.06 & 1.09 & 3.31 & 0.49 \\
\hline HD $25896 \ldots \ldots \ldots . . . .$. & 0.48 & 0.51 & 0.52 & 2.82 & 0.67 \\
\hline HD $26434 \ldots \ldots \ldots . .$. & $\ldots$ & 0.54 & 0.56 & 2.27 & 0.47 \\
\hline HD $26173 \ldots \ldots \ldots . .$. & $\ldots$ & 0.67 & 0.70 & 2.39 & 0.41 \\
\hline SAO $111632 \ldots \ldots$ & 1.55 & 1.54 & 1.58 & 3.05 & 0.23 \\
\hline HD $26323 \ldots \ldots \ldots . .$. & 1.06 & 1.06 & 1.09 & 2.73 & 0.28 \\
\hline SAO $111655 \ldots \ldots . .$. & 1.53 & 1.53 & 1.56 & 3.00 & 0.23 \\
\hline HD $26607 \ldots \ldots \ldots . . .$. & 0.97 & 0.93 & 0.95 & 2.61 & 0.51 \\
\hline HD $26691 \ldots \ldots . . . . .$. & 2.04 & 2.07 & 2.06 & 5.65 & 1.14 \\
\hline HD $26738 \ldots \ldots \ldots . . .$. & 1.11 & 1.11 & 1.13 & 2.77 & 0.32 \\
\hline HD $27063 \ldots \ldots \ldots . .$. & $\ldots$ & $\mathrm{b}$ & c & a & $\mathrm{a}$ \\
\hline HD $27146 \ldots \ldots \ldots . . .$. & 0.50 & 0.46 & 0.47 & 2.51 & 0.87 \\
\hline HD $28856 \ldots \ldots \ldots . . .$. & $\ldots$ & 0.33 & 0.34 & a & a \\
\hline SAO $149765 \ldots \ldots . .$. & 0.89 & 0.91 & 0.92 & 2.77 & 0.35 \\
\hline HD $31900 \ldots \ldots \ldots . . .$. & $\ldots$ & 0.32 & 0.33 & 2.28 & 0.67 \\
\hline HD $34639 \ldots \ldots \ldots . . .$. & $\ldots$ & 0.49 & 0.50 & 3.27 & 0.85 \\
\hline SAO $170502 \ldots \ldots$. & $\ldots$ & 0.46 & 0.48 & 2.08 & 0.48 \\
\hline HD $55718 \ldots \ldots \ldots . . .$. & 0.12 & 0.12 & 0.12 & a & a \\
\hline HD $56073 \ldots \ldots \ldots . . . .$. & 0.43 & 0.40 & 0.42 & 3.30 & 0.94 \\
\hline HD $57336 \ldots \ldots \ldots . .$. & 0.68 & 0.69 & 0.70 & 3.60 & 0.73 \\
\hline HD $59421 \ldots \ldots \ldots . . .$. & 0.97 & 1.02 & 1.03 & 3.11 & 0.41 \\
\hline HD $64033 \ldots \ldots \ldots . . . .$. & b & b & 0.06 & $\mathrm{a}$ & $\mathrm{a}$ \\
\hline
\end{tabular}

TABLE 2-Continued

\begin{tabular}{|c|c|c|c|c|c|}
\hline \multirow[b]{2}{*}{$\begin{array}{l}\text { NAME } \\
(1)\end{array}$} & \multicolumn{3}{|c|}{$A_{V}$} & \multirow[b]{2}{*}{$\begin{array}{l}R_{V} \\
(5)\end{array}$} & \multirow[b]{2}{*}{$\begin{array}{r}\sigma R_{V} \\
(6)\end{array}$} \\
\hline & $\begin{array}{l}\text { Free Fit } \\
\text { (2) }\end{array}$ & $\begin{array}{l}\text { High-Latitude Fit } \\
\text { (3) }\end{array}$ & $\begin{array}{c}1.1 E_{V-K} \\
\text { (4) }\end{array}$ & & \\
\hline HD $80531 \ldots \ldots \ldots . . .$. & 0.31 & 0.30 & 0.31 & 2.39 & 0.87 \\
\hline HD $80840 \ldots \ldots \ldots . . .$. & $\ldots$ & 0.86 & 0.88 & 2.60 & 0.32 \\
\hline HD $85304 \ldots \ldots \ldots . . .$. & $\ldots$ & 0.32 & 0.33 & 2.67 & 0.89 \\
\hline HD $94434 \ldots \ldots \ldots . . .$. & 1.48 & 1.46 & 1.50 & 3.21 & 0.27 \\
\hline HD $94413 \ldots \ldots \ldots . . .$. & $\ldots$ & 1.05 & 1.08 & 2.84 & 0.30 \\
\hline HD $94473 \ldots \ldots \ldots . . .$. & $\ldots$ & 0.25 & 0.28 & 2.27 & 0.90 \\
\hline HD $96237 \ldots \ldots \ldots . .$. & $\ldots$ & 0.49 & 0.48 & 1.44 & 0.46 \\
\hline SAO $179675 \ldots \ldots \ldots$ & $\ldots$ & 0.52 & 0.54 & 3.08 & 0.73 \\
\hline HD 98837 ............. & 0.89 & 0.93 & 0.95 & 2.79 & 0.37 \\
\hline HD $99786 \ldots \ldots \ldots . . .$. & $\ldots$ & 0.78 & 0.77 & 3.12 & 0.49 \\
\hline HD $100359 \ldots \ldots .$. & 1.01 & 0.99 & 1.02 & 2.59 & 0.22 \\
\hline HD $106631 \ldots \ldots . .$. & 0.75 & 0.75 & 0.77 & 2.16 & 0.26 \\
\hline HD $125679 \ldots \ldots \ldots$ & 0.32 & 0.34 & 0.33 & 1.91 & 0.70 \\
\hline HD $139515 \ldots \ldots \ldots$ & $\ldots$ & 0.84 & 0.86 & 3.25 & 0.48 \\
\hline HD $140536 \ldots \ldots . . .$. & $\ldots$ & 0.22 & 0.22 & 2.17 & 0.89 \\
\hline HD $141423 \ldots \ldots \ldots$ & 1.33 & 1.32 & 1.35 & 2.83 & 0.24 \\
\hline HD $141569 \ldots \ldots \ldots$ & 0.55 & 0.53 & 0.54 & 3.64 & 0.90 \\
\hline HD $141657 \ldots \ldots \ldots$ & 0.86 & 0.87 & 0.89 & 2.54 & 0.30 \\
\hline HD $142554 \ldots \ldots \ldots$ & $\ldots$ & 0.75 & 0.72 & 3.57 & 0.62 \\
\hline HD $142863 \ldots \ldots \ldots$ & 1.22 & 1.21 & 1.24 & 2.91 & 0.27 \\
\hline HD $142930 \ldots \ldots \ldots$ & 0.61 & 0.59 & 0.59 & 2.64 & 0.44 \\
\hline HD $143294 \ldots \ldots \ldots$ & & 0.94 & 0.97 & 3.14 & 0.41 \\
\hline HD $145631 \ldots \ldots \ldots$ & 0.84 & 0.79 & 0.80 & 3.82 & 0.53 \\
\hline HD $146931 \ldots \ldots \ldots$ & 0.90 & 0.89 & 0.91 & 3.11 & 0.41 \\
\hline HD $147010 \ldots \ldots \ldots$ & 0.62 & 0.68 & 0.65 & 2.81 & 0.45 \\
\hline HD $153212 \ldots \ldots \ldots$ & 1.22 & 1.27 & 1.31 & 2.55 & 0.33 \\
\hline HD $153605 \ldots \ldots \ldots$ & 1.15 & 1.15 & 1.16 & 2.87 & 0.42 \\
\hline HD $156225 \ldots \ldots \ldots$ & 0.70 & 0.70 & 0.71 & 3.34 & 0.61 \\
\hline HD $156597 \ldots \ldots \ldots$. & 2.16 & 2.11 & 2.16 & 3.22 & 0.18 \\
\hline HD $156822 \ldots \ldots \ldots$ & 0.24 & 0.25 & 0.24 & a & a \\
\hline HD $156912 \ldots \ldots \ldots . .$. & & $\mathrm{b}$ & d & a & a \\
\hline HD $157280 \ldots \ldots \ldots$ & 2.12 & 2.10 & 2.14 & 3.31 & 0.20 \\
\hline HD $157333 \ldots \ldots \ldots$ & 2.24 & 2.30 & 2.31 & 2.95 & 0.15 \\
\hline HD $157512 \ldots \ldots \ldots$. & 0.40 & 0.37 & 0.38 & 3.84 & 1.30 \\
\hline HD $187558 \ldots \ldots \ldots$ & 1.06 & 1.05 & 1.07 & 2.30 & 0.37 \\
\hline HD $187869 \ldots \ldots \ldots$ & 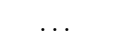 & 0.40 & 0.40 & 2.83 & 1.19 \\
\hline HD $188220 \ldots \ldots \ldots$ & 0.94 & 0.99 & 0.99 & 2.54 & 0.43 \\
\hline HD $188350 \ldots \ldots \ldots$ & 0.43 & 0.44 & 0.44 & 3.55 & 0.94 \\
\hline HD $189115 \ldots \ldots \ldots . . .$. & 1.49 & 1.42 & 1.46 & 5.33 & 1.32 \\
\hline HD $192435 \ldots \ldots \ldots$ & $\ldots$ & 1.12 & 1.11 & 4.56 & 0.77 \\
\hline HD $198772 \ldots \ldots \ldots$ & $\ldots$ & 0.27 & 0.27 & 2.71 & 1.61 \\
\hline HD $199469 \ldots \ldots \ldots$ & $\ldots$ & b & c & ${ }^{a}$ & a \\
\hline HD $201379 \ldots \ldots \ldots$ & & 0.23 & 0.22 & 2.30 & 1.31 \\
\hline HD $203532 \ldots \ldots \ldots$ & 0.89 & 0.96 & 0.96 & 2.78 & 0.31 \\
\hline HD $205037 \ldots \ldots \ldots$ & $\ldots$ & 0.94 & 0.96 & 3.35 & 0.89 \\
\hline HD 207115............... & $\ldots$ & 0.85 & 0.89 & 3.16 & 0.90 \\
\hline HD $210121 \ldots \ldots \ldots$ & 0.85 & 0.88 & 0.87 & 2.13 & 0.22 \\
\hline HD $216039 \ldots \ldots \ldots$ & 0.57 & 0.55 & 0.57 & 2.55 & 0.45 \\
\hline HD $216308 \ldots \ldots \ldots$ & 0.35 & 0.32 & 0.32 & 2.21 & 0.53 \\
\hline
\end{tabular}

Note.-Table 2 is also available in machine-readable form in the electronic edition of the Astrophysical Journal.

${ }^{a} R_{V}$ not accurate for $E_{B-V}<0.10$ mag.

b Data not well fitted by offset power law.

${ }^{c}$ Negative extinction consistent with zero.

${ }^{d}$ Negative extinction indicates possible spectral type error. 


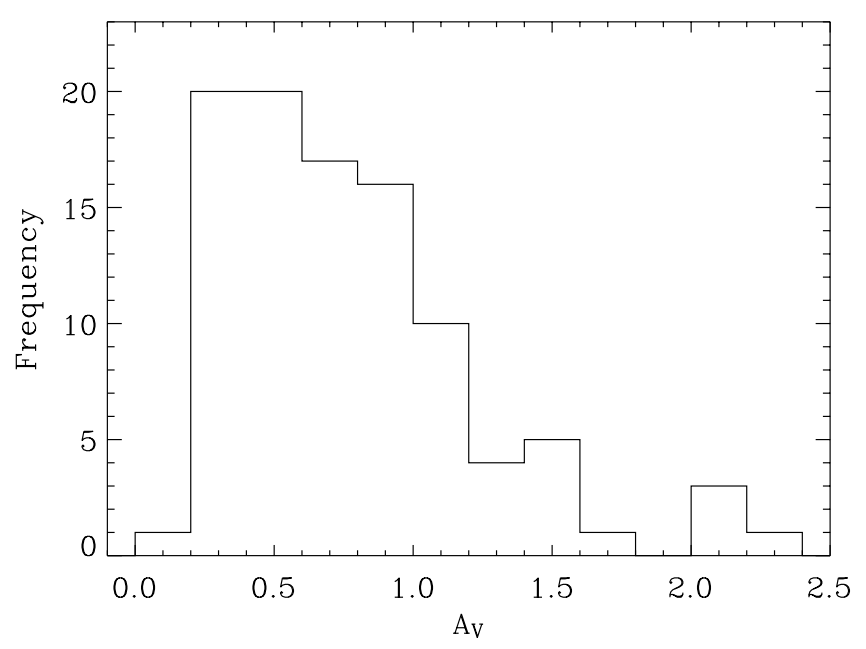

FIG. 1.- Histogram of visual extinction for stars in this program. Values represent the best value of $A_{V}$ for each star (i.e., col. [2] of Table 2 for stars with $L$ photometry and col. [3] for those without).

types of these stars are incorrect. For the high-latitude stars with color excess well fitted by an offset power law, the average parameters of the best-fit normalized infrared extinction curves are

$$
\left\langle\frac{A_{\lambda}}{A_{V}}\right\rangle=(0.46 \pm 0.13) \lambda^{-2.3 \pm 0.5} .
$$

The average value of $\langle\alpha\rangle=2.3$ found at high latitude indicates an average normalized extinction curve that is steeper than the average curve of equation (7). The average high-latitude extinction curve of equation (8) can be used to find the extinction toward high-latitude stars with no $L$ photometry. For these stars, the average high-latitude extinction curve with $A_{V}$ as the only free parameter,

$$
E_{\lambda-V}=A_{V}\left(\left\langle\frac{A_{\lambda}}{A_{V}}\right\rangle-1\right)=A_{V}\left(0.46 \lambda^{-2.3}-1\right)
$$

is fitted to the data with the same fitting algorithm used to fit the general three-parameter form. Values of $A_{V}$ determined by this method are listed in column (3) of Table 2. Figure 1 shows a histogram of the best values of $A_{V}$ for stars in our program, i.e., column (2) of Table 2 for stars with $L$ photometry and column (3) for those without. The average extinction toward all stars in the program is $0.77 \mathrm{mag}$. About one-quarter of the stars in the program have $A_{V}>1$.

The uncertainty in individual values of $\alpha$ is large even for stars with four detected passbands. Compounding the significant uncertainty inherent in fitting a three-parameter function to four data points, the difference between a power law and the data points does not strongly depend on the value of the powerlaw index. In addition, the surface to be minimized forms a steep canyon in $\alpha-A_{V}$ parameter space near the best fit. Therefore, the fitted parameters are somewhat sensitive to the scale of the simplex and the fractional terminating step. Not all best-fit curves pass within the error bars at each point, as expected by allowing the possibility for outliers in the minimization routine, but if the mean curve in equation (8) is forced to pass through $A_{K} / A_{V}$ within standard uncertainty $\sigma_{K}=0.03 \mathrm{mag}$, the uncertainty in the power-law exponent could be as large as $\sigma_{\alpha}=$ $0.42 / A_{V}$. With these cautions in mind, we adopt equation (8) as

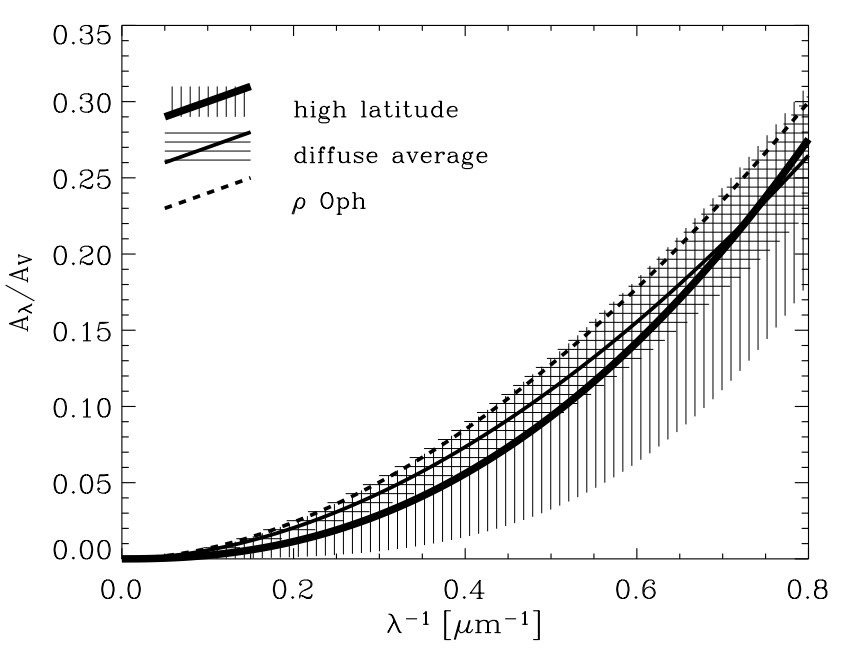

FIG. 2.-Average high-latitude extinction curve (thick solid line) compared to the diffuse ISM average and $\rho \mathrm{Oph}$ (thin solid line and dashed line, respectively; Martin \& Whittet 1990). The range of individual high-latitude lines of sight is indicated by the larger filled area, while the uncertainty in fit parameters over many lines of sight in the diffuse ISM is shown as the smaller filled area.

the "mean high-latitude extinction curve," shown as the thick solid line in Figure 2. The general average of Martin \& Whittet (1990) is shown for comparison as the thin solid line, and the $\rho$ Oph line of sight, representing the dense cloud environment, is shown as the dashed line. The larger filled area shows the range of parameters found at high latitude, while the smaller filled area represents the standard uncertainty in the Martin \& Whittet (1990) parameters.

\subsection{Ratio of Total-to-Selective Extinction}

The preceding section shows that there are two main quantities to be determined from a star's color excess curve: extinction in the reference passband and the shape of the normalized curve expressed as $A_{\lambda} / A_{\text {ref }}$. Traditionally, the extinction curve has been characterized by this ratio not in the infrared but in the blue visual as $A_{B} / A_{V}$. When color excess is normalized to $E_{B-V}$, the extinction curve is measured relative to $A_{V}$ and scaled such that the zero point is at $V$, the slope in the blue visual is 1 , and the long-wavelength limit is the ratio of total-toselective extinction,

$$
R_{V}=-\left[\frac{E_{\lambda-V}}{E_{B-V}}\right]_{\lambda \rightarrow \infty}=\frac{A_{V}}{E_{B-V}} .
$$

Therefore, like finding $A_{V}$ by extrapolating the reddening curve, $R_{V}$ can be determined by extrapolating the normalized reddening curve. Note that while infrared color excess is useful to extrapolate the extinction curve, $R_{V}$ (like $\left.A_{V}\right)$ is not itself an indicator of infrared extinction but is instead a measure of the shape of the normalized curve in the visible,

$$
\frac{A_{B}}{A_{V}}=\frac{1}{R_{V}}+1 .
$$

Normalized absolute extinction outside of the blue visual can be calculated from color excess normalized to $E_{B-V}$ if $R_{V}$ is known, where

$$
\frac{A_{\lambda}}{A_{V}}=\frac{1}{R_{V}} \frac{E_{\lambda-V}}{E_{B-V}}+1 .
$$

In addition, if grain response to environmental conditions is such that the curve shape changes systematically and coherently, as 


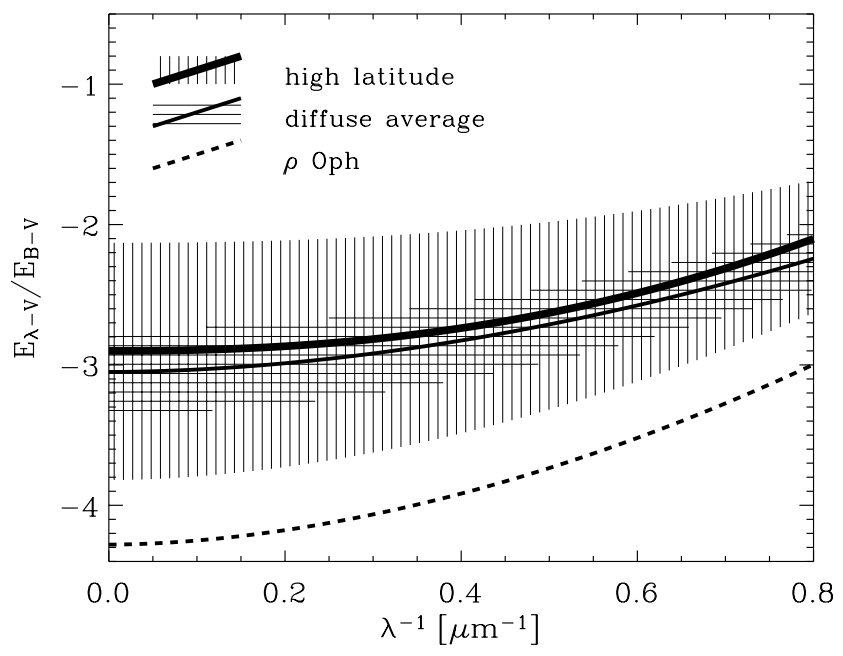

FIG. 3.-Normalized reddening at high latitude (thick solid line) compared to the diffuse ISM average and $\rho$ Oph (thin solid line and dashed line, respectively; Martin \& Whittet 1990). High-latitude reddening is calculated from the average high-latitude extinction curve and $R_{V}=2.9$. Most of the variation in normalized reddening is due to variation in the value of $R_{V}$. The range of $R_{V}$ for individual high-latitude lines of sight is indicated by the larger filled area. The range of values observed toward the LSS is shown as the smaller filled area, representing diffuse lines of sight (He et al. 1995).

has been observed in many regions of the ISM, then the value of $R_{V}$ will characterize the normalized extinction curve shape over a wide wavelength range. This parameter has important widespread usage because it allows the absolute extinction $A_{V}$ to be deduced from the observable color excess $E_{B-V}$.

Column (5) of Table 2 lists $R_{V}=A_{V} / E_{B-V}$ calculated from the best value of $A_{V}$ for each star (i.e., col. [2] for stars with $L$ photometry and col. [3] for those without). Note that for a star with $E_{B-V}<0.10 \mathrm{mag}$, the value of the ratio is overwhelmed by photometric uncertainty in the dividend and divisor. Results from these calculations are omitted from the table. The unweighted mean value of the 94 remaining sight lines at high latitude is

$$
\left\langle R_{V}\right\rangle=2.9
$$

We can express the average NIR color excess normalized to $E_{B-V}$ in terms of $R_{V}$ and the high-latitude extinction curve,

$$
\left\langle\frac{E_{\lambda-V}}{E_{B-V}}\right\rangle=\frac{A_{V}}{E_{B-V}}\left(\left\langle\frac{A_{\lambda}}{A_{V}}\right\rangle-1\right)=2.9\left(0.46 \lambda^{-2.3}-1\right)
$$

shown as the thick solid line in Figure 3. For comparison, the general average curve with $R_{V}=3.05$ representing diffuse lines of sight is shown as the thin solid line, while $\rho$ Oph with $R_{V}=$ 4.28 is shown as the dashed line (Martin \& Whittet 1990).

\subsubsection{Error and Uncertainty in $R_{V}$}

Uncertainty in measurements must be seriously considered in the interpretation of any $R_{V}$ calculation, but uncertainty becomes critical in low-extinction lines of sight. In order to draw meaningful conclusions about the average and range of values of $R_{V}$, this uncertainty must be carefully examined. In this section, we investigate the main sources of error and uncertainty in $R_{V}$ and show that, while the uncertainty of any individual calculation may be large, these calculations on the whole are not systematically skewed to low or high values by that uncertainty.
One method used to extrapolate normalized color excess to its long-wavelength limit is the approximation

$$
R_{V} \simeq 1.1 \frac{E_{V-K}}{E_{B-V}}
$$

shown to be valid for a range of grain models and an accurate representation of data in many different environments (Whittet \& van Breda 1978). We choose this approximation for a detailed investigation of uncertainty so that our results will be valid for any calculation of normalized color excess, the directly observable quantity, and will not depend on a specific model for normalized extinction. In normalized color excess, the principal components and thus the source of any resulting uncertainty or error are measured infrared photometry $(K)$, optical photometry ( $V$ and $B-V)$, and spectral type with intrinsic colors $\left[(V-K)_{0}\right.$ and $\left.(B-V)_{0}\right]$. In the following discussion, we first use standard methods of error propagation to evaluate the effect that uncertainty in each measured component of the calculation has on the result of algebraic combination. More significantly, we then examine the effects of low precision in spectral type, which has a nonlinear effect on the error in associated intrinsic color.

The parameter $R_{V}$ is a dependent quantity that is a function of several measured quantities, $x_{i} \pm \sigma_{i}$. Repeat measurements will form a distribution with standard deviation $\sigma_{R}$. In any estimate of the uncertainty in $R_{V}$, uncertainty in the measured quantities must be propagated through the relationship used to calculate the dependent quantity. In this case, the variance in $R_{V}$ can be approximated using the general formula

$$
\sigma_{R}^{2} \simeq \sum_{i} \sigma_{i}^{2}\left(\frac{\partial R}{\partial x_{i}}\right)^{2}
$$

if the errors are uncorrelated. Because the optical photometry, the NIR photometry, and the spectral type are determined with different instruments on different systems, this assumption is likely to be valid. The result can be expressed as

$$
\left(\frac{\sigma_{R}}{R}\right)^{2}=\frac{\sigma_{V}^{2}+\sigma_{K}^{2}+\sigma_{(V-K)_{0}}^{2}}{E_{V-K}^{2}}+\frac{\sigma_{B-V}^{2}+\sigma_{(B-V)_{0}}^{2}}{E_{B-V}^{2}} .
$$

To apply this equation, consider a model typical of the sight lines in our program: $A_{V}=1.0$ mag and $R_{V}=2.9$, thus $E_{B-V}=$ $0.34 \mathrm{mag}$. Assuming conservative values of the various measurement uncertainties,

$$
\begin{array}{cc}
\sigma_{V} & =0.02, \\
\sigma_{B-V} & =0.02, \\
\sigma_{K} & =0.03, \\
\sigma_{(V-K)_{0}} & =0.05, \\
\sigma_{(B-V)_{0}} & =0.05,
\end{array}
$$

the uncertainty of $R_{V}$ calculated in the model line of sight is $\sigma_{R}=0.5$. Note that the uncertainty in $R_{V}$ is inversely proportional to reddening, so that in this example, $\sigma_{R} \simeq 0.1$ when $E_{B-V}=1.6 \mathrm{mag}$, i.e., when $A_{V}=4.5 \mathrm{mag}$. The values of $\sigma_{R}$ calculated with $\sigma_{K}$ and $\sigma_{E_{B-V}}$ from Table $1, \sigma_{V}=0.02$, and $\sigma_{(V-K)_{0}}=0.02$ are listed in column (6) of Table 2 .

Error propagation calculated in this way is relevant for uncertainty in measurement because of the precision of photometry or the accuracy of intrinsic color curves where the 


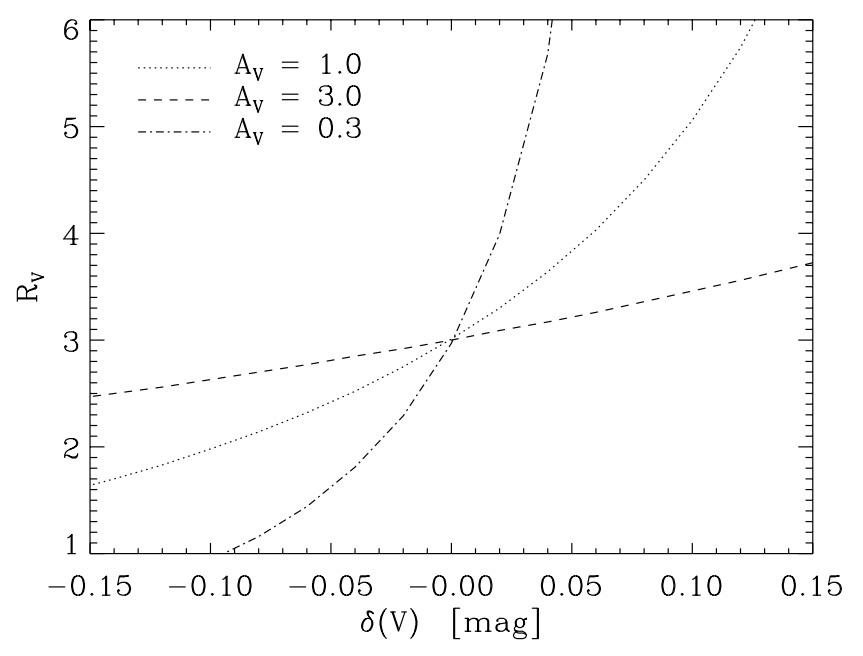

FIG. 4.-Change in $R_{V}$ around $R_{V}=3.0$ due to error in photometry. Three models with different values of $A_{V}$ are shown.

uncertainties are random with a normal Gaussian distribution. The resulting standard deviation indicates the range of possible variation in $R_{V}$. On the other hand, real error in $R_{V}$ may be due to more than random error in measurement, and uncertainty in intrinsic colors may be due to more than just the accuracy of the color curves. For example, the intrinsic colors may not be in the same photometric system as the data. Small inaccuracies in spectral type may yield errors in the intrinsic colors that are relatively large and correlated. In addition, the amount of uncertainty in intrinsic colors is a function of spectral type because the color curves vary in steepness across spectral type. Because infrared intrinsic color curves at B0 V are relatively flat, for example, uncertainty in spectral type around $\mathrm{B} 0 \mathrm{~V}$ will have less effect on the intrinsic color than uncertainty in intrinsic colors of $\mathrm{K}$ stars, where the curves are more steep. In order to investigate such nonlinear effects, we build models of a line of sight where $R_{V}=3.0$. Color pairs are chosen to create models that represent a range of extinction; then various components of the $R_{V}$ calculation are systematically varied over a wide range. The results are plotted as the variation $\delta$ (component) versus $R_{V}$, hereafter called the variation curves.

The simplest effects to consider are variations in photometry. While uncertainty in photometry can be reduced by careful observing techniques, projects that combine photometry from a variety of sources cannot always rule out the possibility of systematic error. Variation in $K$ is the least dangerous, as it simply introduces a reduced added or subtracted component into $R_{V}$. If $K \rightarrow K+\delta K$, then

$$
R_{V} \rightarrow 1.1 \frac{E_{V-K}-\delta K}{E_{B-V}}=R_{V}-\frac{1.1 \delta K}{E_{B-V}}
$$

However, variation in $V$ can be problematic at low extinction because a discontinuity will arise when $\delta V=E_{B-V}$. If $V \rightarrow$ $V+\delta V$, then

$$
R_{V} \rightarrow 1.1 \frac{E_{V-K}+\delta V}{E_{B-V}-\delta V}
$$

Therefore, small errors in the optical photometry can result in huge errors in $R_{V}$ when values of reddening and extinction are small. The effect can be clearly seen in the variation curve of Figure 4, where resulting $R_{V}$ is plotted versus error in $V$ for models with three different values of extinction. Photometric

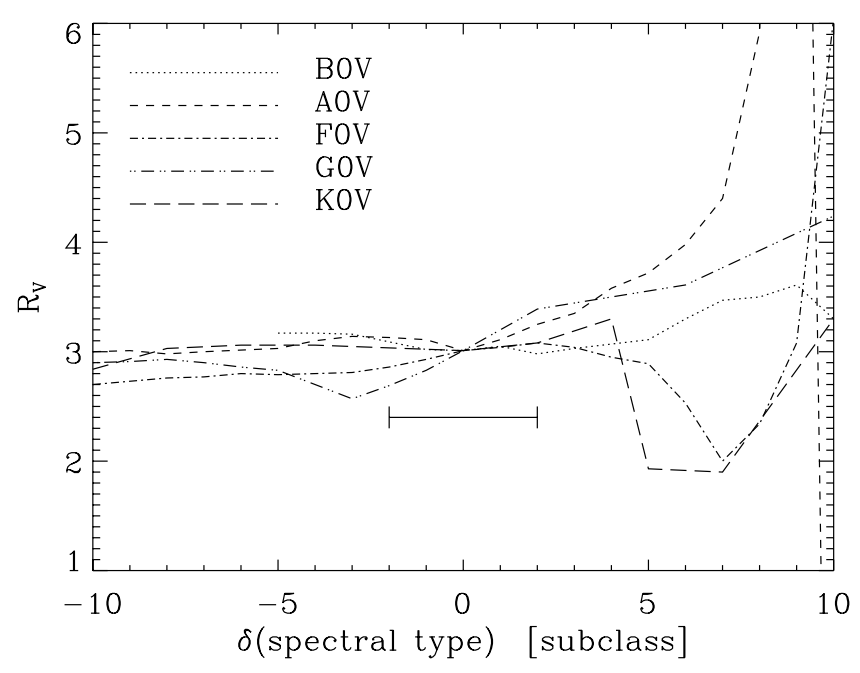

FIG. 5.- Change in $R_{V}$ around $R_{V}=3.0$ due to error in spectral type. Models with $A_{V}=1 \mathrm{mag}$ and five different spectral types are shown. Note that the error in $R_{V}$ is small but biased toward larger values of $R_{V}$ for spectral type errors of two subclasses or less.

error is clearly more significant in the low-extinction model. The effect of error in photometry depends on extinction but not on spectral type. That is, if the extinction and therefore reddening is large, then changes in magnitudes or colors will have less relative effect for all spectral types. It should also be noted that the curves are not symmetric about zero variation; low values of $V$ produce values of $R_{V}$ that are closer to the "real" value of the model than values of $V$ that are too high by the same amount. The significance for our project is that errors in optical photometry are likely to shift to the average of $R_{V}$ to higher values, not lower.

Error in spectral type is much more complicated than error in photometry because intrinsic colors are not linear functions of spectral type. In addition, the infrared $(V-K)_{0}$ and optical $(B-V)_{0}$ intrinsic color curves do not have the same slopes, so error in spectral type contributes differently to infrared and optical color excess, but in a correlated way. If the spectral type itself is wrong, a potentially large error can be introduced into the calculation through the intrinsic colors. Therefore, error in spectral type has different effects on $R_{V}$ for stars of different spectral types. We consider error in one-dimensional spectral type within the dwarf class, error in spectral type near A0 $\mathrm{V}$ for models with a range of extinction, and error in luminosity class.

First, we create models with $A_{V}=1.0 \mathrm{mag}$ and $R_{V}=3.0$ at five spectral types and then vary the spectral type and recalculate $R_{V}$. Figure 5 shows variation in spectral type in integral steps of spectral subclass for models of five dwarf spectral classes: B0 V, A0 V, F0 V, G0 V, and K0 V. All curves are centered at the point of zero variation, that is, at the point of no error in spectral type. For all spectral types considered, if $R_{V}$ is calculated assuming a type that is too early, variation in $R_{V}$ is surprisingly small and for most spectral types, less than $\sigma_{R}$ (eq. [17]) over a wide range of the variation curve. On the other hand, if $R_{V}$ is calculated assuming a spectral type that is late by more than five subclasses, the variation curves are unstable with discontinuities. Of the spectral types considered, B0 V is generally the most stable, followed by $\mathrm{G} 0 \mathrm{~V}, \mathrm{~F} 0 \mathrm{~V}$, and $\mathrm{K} 0 \mathrm{~V}$, with $\mathrm{A} 0 \mathrm{~V}$ being the least stable. In practice, if the spectral type is known to within \pm 2 subclasses, then the error in $R_{V}$ is within \pm 0.2 (except for G0 V, where the error is \pm 0.5 ). Fortunately, this error is on the order of the statistical uncertainty in $R_{V}$ for an $A_{V}=1$ mag model with 


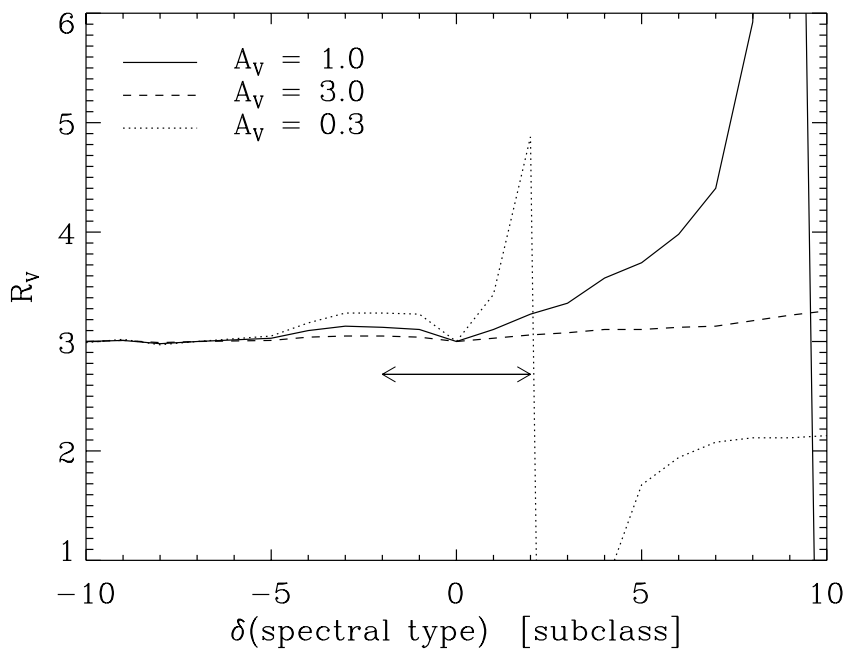

FIG. 6.-Change in $R_{V}$ around $R_{V}=3.0$ due to error in spectral type. Models of A0 V stars with three different values of $A_{V}$ are shown. Errors in spectral type are severe for low-extinction lines of sight.

conservative estimates of random errors. Most relevant to our project, however, is the fact that the error in $R_{V}$ is not biased toward lower values of $R_{V}$. On the contrary, it is most likely that small errors in spectral type will lead to anomalously large calculated values of $R_{V}$. Small values for $R_{V}$ will result only if F0 or G0 types are replaced with spectral types that are too early.

As in the case of photometric variation curves, discontinuities will occur if the intrinsic color overtakes the measured color and leaves small numbers in the denominator of normalized color excess. This situation is more likely to happen if the observed color excess used for normalization, $E_{B-V}$ in our model, is small. Figure 6 shows variation in spectral subclass for A0 V stars with three values of extinction. The $A_{V}=1$ model from Figure 5 is shown as the solid line. The large extinction model $\left(A_{V}=3.0 \mathrm{mag}\right)$ shows no significant error within reasonably expected error in spectral type. However, the low-extinction model $\left(A_{V}=0.3 \mathrm{mag}\right)$ has large variation if the spectral type is only two subclasses late, which may well occur in determining spectral type from photometry or low-resolution spectroscopy (Penprase 1992). As with the error in photometry, the error in spectral type will more quickly introduce spurious values of $R_{V}$ if reddening values are small. The amount of reddening only increases or decreases the amount of error in $R_{V}$, not the direction. Therefore, errors in spectral type around $\mathrm{A} 0 \mathrm{~V}$ for the range of extinction from 0.3 to 3.0 mag are more likely to yield larger values of $R_{V}$ than smaller values of $R_{V}$.

The situation for errors in luminosity class is somewhat different. Sixteen stars in our program have no cataloged luminosity class. On the basis of the small distances that would be implied if late-type stars were dwarfs, we assume stars G and later to be giants. The early-type stars with no cataloged luminosity class are assumed to be dwarfs. To estimate the possible errors that are introduced by these assumptions, we again construct models with $A_{V}=1.0 \mathrm{mag}$ and $R_{V}=3.0$ at five spectral types, but this time we vary the luminosity class before recalculating $R_{V}$. If $R_{V}$ values of models with $R_{V}=3.0$ at $\mathrm{B} 0 \mathrm{III}$, A0 III, F0 III, G0 III, and K0 III are recalculated assuming incorrectly that they are dwarfs, results are mixed, as shown in Table 3. While the error in $R_{V}$ can be large, note that the error can be either positive or negative. In other words, error in luminosity class can yield both high and low values of $R_{V}$ uncorrelated with spectral type. Of the spectral type errors considered, none leads to $R_{V}<2.5$. If the five giant class models are assumed to be dwarfs, the average $R_{V}$ is 3.18 , slightly larger than that actual model average. When stars without a well-determined luminosity class are omitted from our program, the average value for $R_{V}$ is higher by 0.02 . Stars without a well-determined luminosity class make up fewer than one-quarter of the lines of sight in our program with $R_{V}<2.8$. Any systematic offset in spectral type and luminosity class would be apparent as a correlation between $R_{V}$ and spectral type, but none is observed in our program.

Results from our investigation can be used to set a limit on the confidence in $R_{V}$ given reasonable expectations for both measurement uncertainty and possible error. The cutoff of $E_{B-V}>$ $0.1 \mathrm{mag}$ for analysis of $R_{V}$ is justified. Reliable values of $R_{V}$ can be calculated from NIR photometry for low-extinction stars only if the photometry is known to within 0.02 mag and the spectral type is known to within one subclass. On the other hand, the same calculation can be made for high-extinction stars $\left(A_{V}>3 \mathrm{mag}\right)$ with reasonable confidence even if the optical photometry is only known to within $0.1 \mathrm{mag}$ or the spectral type is only known to within several subclasses.

\section{DISCUSSION}

The average high-latitude normalized extinction curve is qualitatively similar to the general average curve of Martin \& Whittet (1990). As shown in Figure 2, the high-latitude curve (thick solid line) is similar in shape to the general average (thin solid line). In addition, when all of the stars in our project are fitted with an adopted curve shape to find the remaining free parameter $A_{V}$, the values of $A_{V}$ that are obtained are equivalent to within $0.1 \mathrm{mag}$ if the general average curve is used instead of the high-latitude extinction curve. Furthermore, values of $A_{V}$ determined by fitting the high-latitude extinction curve are similar to the approximation

$$
A_{V} \simeq 1.1 E_{V-K}
$$

shown in column (4) of Table 2. Extinction calculated with the simple approximation of equation (21) equals the long-wavelength

TABLE 3

Models of Error in $R_{V}$ Due to Error in Luminosity Class

\begin{tabular}{|c|c|c|c|c|c|}
\hline Model Spectral Type & Model $A_{V}$ & Model $R_{V}$ & Assumed Spectral Type & Calculated $R_{V}$ & Error in $R_{V}$ \\
\hline 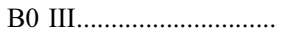 & 1.0 & 3.0 & $\mathrm{~B} 0 \mathrm{~V}$ & 3.1 & +0.1 \\
\hline 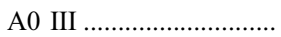 & 1.0 & 3.0 & $\mathrm{~A} 0 \mathrm{~V}$ & 2.8 & -0.2 \\
\hline 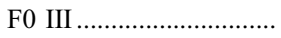 & 1.0 & 3.0 & $\mathrm{~F} 0 \mathrm{~V}$ & 4.0 & +1.0 \\
\hline 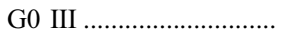 & 1.0 & 3.0 & G0 V & 3.4 & +0.4 \\
\hline 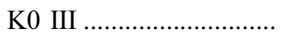 & 1.0 & 3.0 & $\mathrm{~K} 0 \mathrm{~V}$ & 2.6 & -0.4 \\
\hline 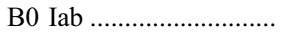 & 1.0 & 3.0 & $\mathrm{~B} 0 \mathrm{~V}$ & 2.9 & -0.1 \\
\hline 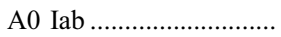 & 1.0 & 3.0 & $\mathrm{~A} 0 \mathrm{~V}$ & 2.9 & -0.1 \\
\hline
\end{tabular}


limit of a fitted offset power law to within $5 \%$ on average and usually to within $0.1 \mathrm{mag}$. The approximation factor of 1.1 is equivalent to

$$
\left(1-\frac{A_{K}}{A_{V}}\right)^{-1}
$$

or 1.10 for the general average curve and 1.08 for the highlatitude curve. Even an exponent of $\alpha=3.0$ leads to an approximation factor that rounds to 1.1 .

Yet while the high-latitude extinction curve is similar to the general average curve in the solar neighborhood, we assert that the distinctions between the two curves are significant. The larger filled area in Figure 2 represents the range of best-fit extinction curves found in our program. For comparison, the uncertainty in the fit parameters of the Martin \& Whittet (1990) general average is shown by the smaller filled area. The highlatitude curves include many lines of sight with relatively less extinction at long wavelengths and progressively more extinction toward shorter wavelengths. In other words, many of the high-latitude curves rise more steeply than the general average. Even though all three curves in Figure 2 appear similar, the average value of the high-latitude power-law index $(\alpha=$ 2.3) is steeper than the range of the Martin \& Whittet (1990) diffuse average $(\alpha=1.85 \pm 0.10)$ and steeper than $\rho$ Oph $(\alpha=1.82)$. The variation among individual extinction curves at high latitude is greater than the difference between the highlatitude average and the general average curve, but 55 of the 64 curves are steeper than the general average. If only the 22 fitted stars with $A_{V}>1 \mathrm{mag}$ are considered, the average value of the power-law index is slightly less at $\alpha=2.2$, but all 22 curves are steeper than the Martin \& Whittet (1990) average. Our program of high-latitude sight lines can also be compared to a study of 154 highly reddened luminous stars in the southern Milky Way (LSS; He et al. 1995). Extinction toward the bright, early-type stars of the LSS is more than the extinction observed in our program but is generally due to the diffuse ISM along long lines of sight. The LSS represents an average against which we can compare the specific translucent sight lines of our program. When the same fitting procedure used in our study is applied to the LSS data in He et al. (1995), the average value of the power-law index is $\alpha=1.74 \pm 0.40$, consistent with the general average for the diffuse ISM and less steep than the average for high-latitude sight lines in this program. Because normalized extinction curves are independent of the total column density of dust, any variations in normalized extinction curves are due to variations in the physical or optical properties of the dust.

In order to compare the data in our program to common general averages, we have normalized infrared extinction to the visual. Normalizing the NIR extinction curve to the visual, however, can mask the true source of variations. Spatial variations in the normalized NIR extinction curve may be due to the ratio of NIR-to-visual extinction, the shape of the extinction curve within the infrared, or a combination of both effects. To investigate the reason that the NIR extinction curve normalized to $V$ is steeper at high latitude, we reexpress the power-law form of the extinction curve in terms of curve shape normalized to $K$,

$$
\frac{A_{\lambda}}{A_{V}}=\frac{A_{K}}{A_{V}}\left(\frac{A_{\lambda}}{A_{K}}\right)=\frac{A_{K}}{A_{V}}\left(e_{K} \lambda^{-\alpha}\right) .
$$

When the NIR extinction is normalized to an NIR passband, the normalizing scale factor $e_{K}$ must be related to the value of the power law at that passband,

$$
e_{K}=\frac{1}{\lambda_{K}^{-\alpha}}=\frac{1}{2.2^{-\alpha}} .
$$

We can therefore express the curve normalized to $V$ with just two parameters: the scaling ratio $A_{K} / A_{V}$ and the power-law index $\alpha$. In this new formulation, we rewrite the high-latitude extinction curve of equation (8) as

$$
\frac{A_{\lambda}}{A_{V}}=\frac{A_{K}}{A_{V}}\left(\frac{\lambda}{2.2}\right)^{-\alpha}=0.075\left(\frac{\lambda}{2.2}\right)^{-2.3}
$$

and general average curve of Martin \& Whittet (1990) from equation (7) as

$$
\frac{A_{\lambda}}{A_{V}}=0.093\left(\frac{\lambda}{2.2}\right)^{-1.85} .
$$

The exponent in the power law indicates the shape of the NIR extinction curve itself, while the scaling ratio indicates the amount of NIR extinction relative to visual extinction. By expressing the curve shape in this form, we can conclude that the differences between the average high-latitude curve and the general average curve are due to a steeper NIR curve at high latitude as well as reduced NIR extinction relative to visual extinction. In other words, both parameters show that the high-latitude extinction curve becomes steeper toward shorter wavelengths. Of the two effects, variation within the NIR passbands may be dominant. The average ratio of $K$ extinction relative to visual extinction can be calculated directly from $A_{V}$ in Table 2 and the color excess data as

$$
\frac{A_{K}}{A_{V}}=\left\langle\frac{E_{K-V}}{A_{V}}\right\rangle+1 .
$$

The average value of the ratio of NIR-to-visual extinction calculated by this method is $\left\langle A_{K} / A_{V}\right\rangle=0.07 \pm 0.03$, consistent with the value calculated directly from the average highlatitude extinction curve.

Further evidence of significant differences between highlatitude extinction and the average diffuse ISM can be found in closer analysis of the parameter $R_{V}$. If all extinction curves scaled simply with column density of the foreground dust, then values of $R_{V}$ would be uniform. Indeed, $R_{V}$ has been measured to be near 3.05 throughout the diffuse ISM. In some lines of sight, however, the extinction curves do not scale simply with column density. Because the extinction efficiency of dust grains peaks for sizes on the order of the wavelength, variations in the grain size distribution will cause variations in normalized extinction curve shape and thus variation in values of $R_{V}$ (see Whittet 2003 for review). As shown in equation (11), a flatter normalized extinction curve means relatively less extinction in the $B$ passband and therefore larger values of $R_{V}$. Steeper normalized extinction curves, on the other hand, have relatively more extinction in the $B$ passband and therefore smaller values of $R_{V}$. Systematic shifts in the grain size distribution will lead to a coherent change of the shape of the normalized extinction curve across the entire wavelength range. Such coherent changes in extinction curve shape have been observed, for example, in the correlation between $R_{V}$ 


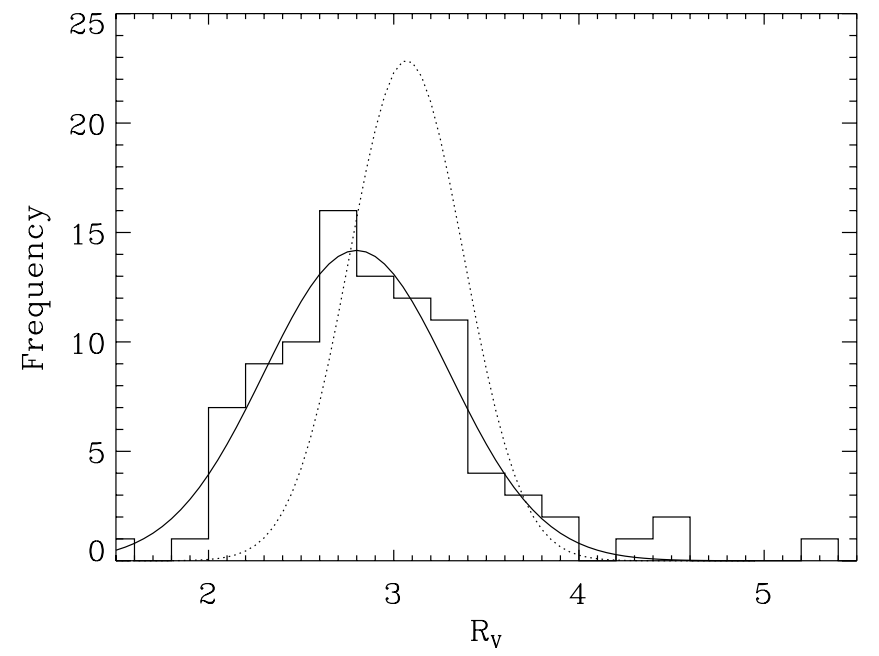

FIG. 7.- Histogram of high-latitude $R_{V}$. The solid curve represents a Gaussian normalized to the data with $R_{V}=2.8 \pm 0.5$. A Gaussian distribution with equivalent normalization and the LSS value of $R_{V}=3.07 \pm 0.31$ (He et al. $1995)$ is shown as the dotted curve and represents the diffuse ISM.

and variations in the extinction curve from the visible out into the ultraviolet (e.g., Cardelli et al. 1989). In dense clouds, values of $R_{V}$ are significantly larger than the diffuse average not because of a simple increase of $A_{V}$ in the fraction

$$
R_{V}=\frac{A_{V}}{E_{B-V}},
$$

but because of the relative lack of small grains in those lines of sight as a result of coagulation. Conversely, small values of $R_{V}$ are attributed to a relative enhancement of small grains. One of the stars in this program, HD 210121, is located behind a cloud with an abundance of small dust grains causing a very small value of $R_{V}$ and one of the steepest UV extinction curves observed (Larson et al. 1996, 2000).

We have found that the high-latitude NIR extinction, on average, is steeper toward shorter wavelengths and that the amount of visual extinction is elevated with respect to the longer NIR wavelengths. From these observations, it might be expected that the extinction curve shortward of the $V$ passband toward $B$ would be steeper as well. Indeed, we find that the high-latitude values of $R_{V}$ are not entirely consistent with the range of values found in the diffuse ISM. A histogram of $R_{V}$ values in our program is shown in Figure 7. While $R_{V}$ is nearly the same on average at high latitude as that in the diffuse ISM, our program includes several stars with $R_{V}$ as low as 2, unusually small values for this parameter. Omitting the five sight lines with $R_{V}>$ 4, the average value of $R_{V}$ for the rest of the program is $2.8 \pm 0.5$. A Gaussian curve with an average of $R_{V}=2.8$ and a standard deviation of 0.5 normalized to the number of sight lines is plotted as the solid curve in Figure 7. The range of $R_{V}$ at high latitude can be compared to the range of $R_{V}$ toward the LSS using observations and methods similar to our project (He et al. 1995). The parameter $R_{V}$ toward the LSS has an average value of $\left\langle R_{V}\right\rangle=$ $3.07 \pm 0.31$, representative of the value of $R_{V}$ in the diffuse ISM. The dotted curve in Figure 7 is a Gaussian centered around the LSS average value of $R_{V}$ and normalized to the number of stars in this program. In comparison, the distribution of values of $R_{V}$ for high-latitude sight lines has a much broader range with a distinct shift toward smaller values of $R_{V}$. The distribution is not entirely symmetric around its peak and includes a few clouds with large values of $R_{V}$, more similar to values found in dense clouds. LSS sight lines have $R_{V}$ values that range from 2.6 to 4.6 , but only $2 \%$ have $R_{V}$ values less than 2.8 . In contrast, $47 \%$ of the program stars presented in this paper have low values of $R_{V}<2.8$. The high-latitude environment may contain more than one distinct group of clouds, such as one group with $R_{V} \sim 3$, a few with large $R_{V}$, and a separate group with significantly small values of $R_{V}$. Low-extinction clouds such as those in our program would represent a small component of the total $R_{V}$ observed over long lines of sight in the diffuse ISM and would be difficult to distinguish from several magnitudes of $R_{V} \sim 3$ extinction.

The range of individual normalized reddening curves is indicated by the larger of the filled areas in Figure 3, and the average high-latitude reddening curve (eq. [14]) is shown with a thick solid line. For comparison, the diffuse ISM average curve is shown with a thin solid line. The smaller filled area indicates the range of $R_{V}=3.07 \pm 0.31$ found toward the LSS by $\mathrm{He}$ et al. (1995), representative of the range found throughout the diffuse ISM. The range of long-wavelength intercept values shows that values of $R_{V}$ at high latitude span a larger range than values of $R_{V}$ in the diffuse ISM. Representing the dense cloud environment, $\rho \mathrm{Oph}$ is shown as the dashed line. Note that while the normalized NIR extinction curves in the diffuse ISM and in the dense cloud environment are nearly universal (see Fig. 2), the normalized color excess curves in Figure 3 are very different because of the different values of $R_{V}$. In contrast, $R_{V}$ at high latitude may be weakly correlated with the shape of the normalized NIR extinction curve. The average value of the power-law index for stars with $R_{V}<3.0$ is $\alpha=2.40$, while the average for stars with $R_{V}>3.0$ is $\alpha=2.12$, indicating that lines of sight with small values of $R_{V}$ have steeper extinction curves in the infrared. A trend of increasing $R_{V}$ with increasing $A_{V}$ is expected if and only if increased extinction, such as that observed toward $\rho$ Oph, leads to a relative decrease of small grains in the foreground cloud. For lines of sight in our program, there is only a slight trend between $R_{V}$ and $A_{V}$, as shown in Table 4 . The mean values of $R_{V}$ in all extinction ranges are equal within the uncertainty in the mean, but the lowest extinction lines of sight in this project have slightly smaller $R_{V}$ than the highest extinction lines of sight. The lack of a strong trend between $R_{V}$ and $A_{V}$ shows that low values of $R_{V}$ in high-latitude translucent clouds are not simply due to small numbers in the ratio calculations. We conclude that standard assumptions of the universal average extinction curve and $R_{V} \simeq 3$ are more applicable at high latitude to lines of sight where $A_{V}>1.2 \mathrm{mag}$.

The population of high-latitude lines of sight with small values of $R_{V}$ may represent a population that has not been observed in the Galactic plane. The "enhanced small grain" ISM observed in high-latitude clouds is not simply "average dust" with an extra component of small grains. If that were true, the infrared extinction would be consistent with the general average. The values of $e$ and $\alpha$ that characterize the NIR extinction curve are uniform across a range of environmental conditions, including

TABLE 4

Possible $R_{V}$ Dependence on $A_{V}$

\begin{tabular}{crc}
\hline \hline \multicolumn{1}{c}{$A_{V}$} & $n$ & $R_{V}$ \\
\hline$<0.50 \ldots \ldots \ldots \ldots \ldots \ldots \ldots$. & 28 & $2.70 \pm 0.66$ \\
$0.50 \rightarrow 0.99 \ldots \ldots \ldots \ldots \ldots$. & 41 & $2.94 \pm 0.50$ \\
$1.00 \rightarrow 1.50 \ldots \ldots \ldots \ldots \ldots$ & 17 & $3.06 \pm 0.75$ \\
$>1.50 \ldots \ldots \ldots \ldots \ldots \ldots \ldots$. & 8 & $3.39 \pm 0.93$ \\
\hline
\end{tabular}


dense clouds (Martin \& Whittet 1990). The distinct values of $e$ and $\alpha$ found at high latitude indicate that the relative abundance of larger dust grains causing extinction at long wavelengths is less than the relative abundance common to both the diffuse ISM and dense cloud environments. If $R_{V}$ is an indicator of the slope of the blue visual extinction curve, then $R_{K}$ defined as

$$
R_{K}=\frac{A_{K}}{E_{J-K}}
$$

is an indicator of the average slope of the extinction curve in the NIR. The value of $R_{K}$, like the functional form of the NIR extinction curve itself, has been found to be universal with a common value of $0.54 \pm 0.03$ in both the diffuse ISM and the $\rho$ Oph dense cloud environment (Martin \& Whittet 1990). The average value of $R_{K}=0.45$ at high latitude is less than the ISM universal value, and the high-latitude average drops to 0.41 if the lines of sight with $A_{V}<0.5$ are omitted. Together with the low values of $R_{V}$, the larger average value of the power-law index $\alpha$, and the reduced value of the ratio $A_{K} / A_{V}$, this low value of $R_{K}$ confirms that the high-latitude extinction curve is steeper per unit extinction from NIR to optical wavelengths. We conclude that a significant fraction of high-latitude lines of sight have a systematic shift toward small dust grains.

The recently released 2MASS data set provides a unique opportunity to further search the high-latitude translucent ISM for the extinction signatures of enhanced small-grain abundance. The wealth of photometric data in 2MASS opens the possibility that extinction curve shape may be mapped over the entire high-latitude sky. Several points demonstrated in this paper must be remembered when using 2MASS data to calculate extinction. First, with only three passbands, it will not be possible to simultaneously measure the amount of extinction $A_{\lambda}$ and the two parameters that characterize the shape of the normalized extinction curve, $e_{K}$ and $\alpha$, for example. It will be possible to measure a reliable curve-shape indicator such as $R_{K}$ if and only if the spectral type of the star is known and appropriate, accurate intrinsic colors are chosen. We have shown that calculations of normalized reddening may have large, asymmetrical errors when calculated for low-extinction lines of sight, especially if the spectral types are not well determined. The 2MASS data may allow us to find more moderate-extinction lines of sight at high latitude where calculations will be most accurate. Characterization of the extinction curve relative to the visual passband, while keeping with tradition, requires good optical photometry and colors and introduces the possibility of data mismatch. In this paper, we have shown that systematic changes in the grain size distribution toward smaller grains cause variations in the NIR extinction curve that mirror similar changes in the visible. Therefore, NIR photometry can be used alone to characterize the high-latitude extinction curve and grain size distribution.

This paper uses observations made at the South African Astronomical Observatory (SAAO). We thank D. Kilkenny for unpublished data and assistance at SAAO. This research makes use of data products from the Two Micron All Sky Survey, which is a joint project of the University of Massachusetts and the Infrared Processing and Analysis Center/California Institute of Technology, funded by NASA and NSF. This research also makes use of the SIMBAD database, operated at CDS, Strasbourg, France. This research is supported by NASA grant NAG5-7598 to Rensselaer Polytechnic Institute and an AAS Small Research Grant to K. Larson.
Arce, H. G., \& Goodman, A. A. 1999, ApJ, 512, L135

Bessell, M. S., \& Brett, J. M. 1988, PASP, 100, 1134

Burstein, D., \& Heiles, C. 1982, AJ, 87, 1165

Cardelli, J. A., Clayton, G. C., \& Mathis, J. S. 1989, ApJ, 345, 245

Carter, B. S. 1990, MNRAS, 242, 1

Cutri, R. M., et al. 2003, Explanatory Supplement to the 2MASS All Sky Data Release, http://www.ipac.caltech.edu/2mass/releases/allsky/doc/explsup.html

Désert, F. X., Bazell, D., \& Boulanger, F. 1988, ApJ, 334, 815

Franco, G. A. P. 1988, A\&AS, 74, 73

Glass, I. S. 1974, Mon. Notes Astron. Soc. South Africa, 33, 53 1997, Mon. Notes Astron. Soc. South Africa, 56, 110

Hartley, M., Tritton, S. B., Manchester, R. N., Smith, R. M., \& Goss, W. M. 1986, A\&AS, 63, 27

Hartmann, D., Magnani, L., \& Thaddeus, P. 1998, ApJ, 492, 205

He, L., Whittet, D. C. B., Kilkenny, D., \& Spencer Jones, J. H. 1995, ApJS, 101,335

Larson, K. A., Whittet, D. C. B., \& Hough, J. H. 1996, ApJ, 472, 755

Larson, K. A., Wolff, M. J., Roberge, W. G., Whittet, D. C. B., \& He, L. 2000, ApJ, 532, 1021

Magnani, L., Blitz, L., \& Mundy, L. 1985, ApJ, 295, 402

Magnani, L., Hartmann, D., Holcomb, S. L., Smith, L. E., \& Thaddeus, P. 2000, ApJ, 535, 167

\section{REFERENCES}

Magnani, L., \& Onello, J. S. 1995, ApJ, 443, 169

Martin, P. G., \& Whittet, D. C. B. 1990, ApJ, 357, 113

Mermilliod, J.-C., \& Mermilliod, M. 1994, Catalogue of Mean UBV Data on Stars (New York: Springer)

Penprase, B. E. 1992, ApJS, 83, 273

Press, W. H., Teukolsky, S. A., Vetterling, W. T., \& Flannery, B. P. 1994, Numerical Recipes: The Art of Scientific Computing (2nd ed.; New York: Cambridge Univ. Press)

Reach, W. T., Koo, B.-C., \& Heiles, C. 1994, ApJ, 429, 672

Reach, W. T., Wall, W. F., \& Odegard, N. 1998, ApJ, 507, 507

Schlegel, D. J., Finkbeiner, D. P., \& Davis, M. 1998, ApJ, 500, 525

Schmidt-Kaler, T. 1982, in Landolt-Börnstein: Numerical Data and Functional Relationships in Science and Technology, ed. K. Schaifers \& H. H. Voigt (New York: Springer)

van Dishoeck, E. F. 1994, in ASP Conf. Ser. 58, First Symposium on the Infrared Cirrus and Diffuse Interstellar Clouds, ed. R. M. Cutri \& W. B. Latter (San Fransciso: ASP), 319

Wegner, W. 1994, MNRAS, 270, 229

Welty, D. E., \& Fowler, J. R. 1992, ApJ, 393, 193

Whittet, D. C. B. 2003, Dust in the Galactic Environment (London: IOP)

Whittet, D. C. B., \& van Breda, I. G. 1978, A\&A, 66, 57

1980, MNRAS, 192, 467 